\title{
Sु \\ Langevin model for real-time Brownian dynamics of interacting nanodefects in irradiated metals
}

\author{
S. L. Dudarev, ${ }^{1}$ M. R. Gilbert, ${ }^{1,2}$ K. Arakawa,,${ }^{3,4,5}$ H. Mori, ${ }^{3}$ Z. Yao, ${ }^{2,6}$ M. L. Jenkins, ${ }^{2}$ and P. M. Derlet ${ }^{7}$ \\ ${ }^{1}$ EURATOM/CCFE Fusion Association, Culham Centre for Fusion Energy, Abingdon, Oxfordshire OX14 3DB, United Kingdom \\ ${ }^{2}$ Department of Materials, University of Oxford, Parks Road, Oxford OX1 3PH, United Kingdom \\ ${ }^{3}$ Research Center for Ultra-High Voltage Electron Microscopy, Osaka University, 7-1 Mihogaoka, Ibaraki, Osaka 567-0047, Japan \\ ${ }^{4}$ Okinawa Institute of Science and Technology, Kunigami, Okinawa 904-0411, Japan \\ ${ }^{5}$ CREST, JST, 5 Sanbancho, Chiyoda-ku, Tokyo 102-0075, Japan \\ ${ }^{6}$ Department of Mechanical and Materials Engineering, Queen's University, Nicol Hall, \\ 60 Union Street, Kingston, Ontario, Canada K7L $3 N 6$ \\ ${ }^{7}$ Condensed Matter Theory Group, Paul Scherrer Institut, CH-5232 Villigen PSI, Switzerland
}

(Received 18 March 2010; published 15 June 2010)

\begin{abstract}
In situ real-time electron microscope observations of metals irradiated with ultrahigh-energy electrons or energetic ions show that the dynamics of microstructural evolution in these materials is strongly influenced by long-range elastic interactions between mobile nanoscale radiation defects. Treating long-range interactions is also necessary for modeling microstructures formed in ex situ high-dose-rate ion-beam irradiation experiments, and for interpolating the ion-beam irradiation data to the low-dose-rate limit characterizing the neutron irradiation environments of fission or fusion power plants. We show that simulations, performed using an algorithm where nanoscale radiation defects are treated as interacting Langevin particles, are able to match and explain the real-time dynamics of nanodefects observed in in situ electron microscope experiments.
\end{abstract}

DOI: 10.1103/PhysRevB.81.224107

PACS number(s): 61.72.-y, 61.80.-x, 61.82.Bg

\section{INTRODUCTION}

Recent in situ electron microscope observations, providing real-time visualization of dynamics of defects produced by ultrahigh-energy electron irradiation, ${ }^{1-3}$ or showing microstructural evolution occurring under ion beam ${ }^{4-6}$ irradiation, have revolutionized our understanding of how properties of metals and alloys change in the extreme radiation and thermal environments of a fission or a fusion power plant. The key feature of in situ electron microscopy is its ability to exhibit the time-dependent dynamics of migration, interaction, and transformation of radiation defects, and to visualize the entire complexity of evolving defect and dislocation networks. For example, in situ electron microscope observations provided evidence of violation of the Burgers vector conservation law for dislocations on the nanoscale. ${ }^{1}$ This gave a vital clue needed for modeling microscopic processes responsible for the formation of unusual high-temperature dislocation structures in iron, ${ }^{7,8}$ and for explaining the origin of the loss of strength of ferritic-martensitic steels ${ }^{9}$ at high temperatures exceeding $500{ }^{\circ} \mathrm{C}$.

The development of in situ electron microscope techniques was partially stimulated by the application of largescale molecular dynamics (MD) simulations to modeling mobile defects and clusters of defects (for example, nanodislocation loops) in iron and other metals. ${ }^{10-15}$ A hypothesis stating that clusters of point defects play a significant part in microstructural evolution of irradiated materials was proposed in 1990s within the framework of the "production bias" radiation damage model. ${ }^{16}$ However, it is only recently that in situ electron microscope observations ${ }^{1-6}$ confirmed the fact that mobile and immobile clusters of point defects form an integral part of the microstructure of an irradiated material.
Somewhat surprisingly, interpreting in situ real-time electron microscope observations remains genuinely problematic. The ten orders of magnitude mismatch between the nanosecond $\left(10^{-9} \mathrm{~s}\right)$ time scale accessible to an MD simulation, ${ }^{10-15}$ and the $10-1000$ s time scale of a typical in situ electron microscope observation, ${ }^{1-6}$ impedes meaningful quantitative analysis. The need to develop a model, with which real-time observations could be simulated and interpreted, does not only stem from the fact that electron microscopy per se is a highly quantitative technique for characterizing materials. ${ }^{17,18}$ Recently, the question about how to model, in real time, the evolution of an ensemble of mobile interacting radiation defects has been brought into focus by the rapidly growing applications of ion-beam sources to simulating neutron irradiation damage effects in fission and fusion materials.

In situ electron microscope observations visualize the dynamics of microstructure corresponding to the limit of high irradiation dose rates, approaching $10^{-3} \mathrm{dpa} \mathrm{s}^{-1}$ ( $\sim 80$ dpa per $24 \mathrm{~h})$ for the ultrahigh-voltage electron irradiation case, ${ }^{1-3}$ and $6 \times 10^{-4} \mathrm{dpa} \mathrm{s}^{-1}$ to $8 \times 10^{-4} \mathrm{dpa} \mathrm{s}^{-1}$ $(\sim 50-70$ dpa per $24 \mathrm{~h})$ for the in situ ion-beam irradiation case. $^{4-6}$ These dose rates are similar to the $10 \mathrm{dpa}$ per $24 \mathrm{~h}$ to 100 dpa per $24 \mathrm{~h}$ range of dose rates characterizing irradiation conditions in ex situ ion-beam facilities. ${ }^{19}$ In situ electron microscopy and ex situ ion-beam irradiation experiments generate similar microstructures, corresponding to a similar range of high irradiation dose rates. These dose rates are several orders of magnitude higher than the rates associated with the irradiation environment of a fission nuclear reactor, ${ }^{20}$ an accelerator-driven system such as the International Fusion Materials Irradiation Facility (IFMIF) (Ref. 21) or a fusion power plant. ${ }^{22}$

Is there a fundamental difference between microstructures formed in the limits of low and high dose rates? The density 
of defects generated by irradiation in a unit volume of the material per unit time is the main quantity distinguishing defect production under intense high-dose-rate ion-beam or ultrahigh-voltage electron irradiation, and under low-doserate neutron irradiation. This quantity is much higher for high-energy electron or ion-beam irradiation than for neutron irradiation. Since the frequency of interaction events in an ensemble of moving particles is proportional to the square of the density of particles, one should expect that various phenomena associated with the presence of interaction between radiation defects should be more pronounced in the limit of high irradiation dose rate, and should be readily seen in in situ electron microscope experiments. Indeed, there is experimental evidence for the effect of dose rate on microstructural evolution of irradiated materials. ${ }^{23-25}$

In situ electron microscope observations show that simultaneous, as opposed to sequential, production of mobile defects at high irradiation dose rates results in microstructural evolution that is influenced by the "collective" dynamical events involving correlated motion of several defects, leading to the formation of defect rafts, coalescence of defects, and the eventual self-organization and spatial ordering of defects. All these phenomena are routinely seen in in situ electron microscope experiments, ${ }^{1-6}$ suggesting that interaction between radiation defects does play a significant part in the dynamics of microstructural evolution in the limit of high irradiation dose rates.

The fact that elastic interactions between defects might affect microstructural evolution of a material under irradiation was noted by Hudson et al. ${ }^{26,27}$ who investigated, using kinetic Monte Carlo simulations, the evolution of ensembles of interacting defects. The kinetic Monte Carlo model developed by Hudson et al. ${ }^{27}$ treated the effect of elastic forces through the use of hopping probabilities biased by the spatially dependent elastic fields. The study showed that while the effect of elastic forces on the evolution of ensembles of one-dimensionally migrating dislocation loops was indeed significant, ${ }^{26}$ in agreement with earlier MD predictions showing that vacancies pin the motion of glissile dislocation loops, ${ }^{28,29}$ the elastic interactions between threedimensionally migrating defects do not appear to have an appreciable effect on microstructural evolution. ${ }^{27}$ For the treatment of a general case of long-term microstructural evolution, further work is clearly needed to elucidate the role of elastic interactions between the defects. Recent implementations of accelerated kinetic Monte Carlo algorithms for simulating the accumulation of radiation damage ${ }^{30,31}$ are however based on the assumption that defects perform unbiased (by internal elastic fields) three-dimensional migration in the material, and interactions between defects are described as short-range inelastic "collisions." These simulations do not include the treatment of long-range elastic forces acting between the defects, and between defects and dislocations, and moreover do not take into account the effects of onedimensional Brownian motion of prismatic dislocation loops often observed experimentally. ${ }^{1-6}$

In this paper, we describe a possible alternative (to kinetic Monte Carlo) approach to simulating the long time-scale evolution of radiation-induced microstructures. The development of this approach has been stimulated by the fact that understanding the microscopic mechanisms driving microstructural evolution, and matching simulations to experiment, requires modeling particular microscopic realizations of evolving defect structures, like those observed by in situ electron microscopy. Indeed, certain properties of an irradiated material, for example, its fracture toughness or thermal conductivity, depend on the statistical characteristics of irradiation-induced microstructure involving many defects and dislocations, and hence represent self-averaging quantities. At the same time, validating a microstructural evolution model requires understanding the dynamics of interaction between radiation defects, and comparing the results of simulations with local experimental observations, often involving only a few (e.g., two or three) defects, where no statistical ensemble averaging is possible.

Bearing this in mind, in the approach described below we treat defects as interacting objects satisfying a set of linked Langevin equations of motion. It is known that in terms of ensemble averaged quantities, solutions of linked Langevin equations in the strong friction (overdamped) limit are equivalent to the solutions of a multidimensional diffusion equation, ${ }^{32,33}$ and hence they are equivalent to solutions found using kinetic Monte Carlo simulations. ${ }^{27}$ The advantage offered by the Langevin-equations-based treatment is that integrating stochastic differential equations for an ensemble of interacting objects is computationally no more difficult than integrating them for an ensemble of noninteracting particles.

There are further obvious computational advantages offered by the similarity between the Langevin and the MD integration algorithms, like the ease of parallelizing the method. Also, by solving the Langevin equations we explicitly follow the trajectories of migrating defects ${ }^{34,35}$ corresponding to the initial conditions defined by experimental observations whereas kinetic Monte Carlo algorithm ${ }^{27,31}$ operates on the logarithmic time scale, which speeds up calculations but makes it more difficult to compare simulations with observations. Hence, the modes of microstructural evolution predicted by the Langevin dynamics model can be matched and verified explicitly against real-time in situ electron microscope experiments. Furthermore, the speed of the integration algorithm for Langevin dynamics is entirely independent of whether the defects migrate three or one dimensionally. One can easily apply the method to the treatment of either limit, with the potential for generalization to modeling reactions between the defects and between dislocations and the defects.

The paper is organized as follows. We start from giving a brief summary of in situ electron microscope observations, which show evidence of the significant part played by the interaction between mobile radiation effects. We then introduce the simulation method, describe how to treat long-range interactions between defects (the "Langevin particles"), and compare the simulated trajectories of defects with experimental observations. Finally, we discuss effects of interaction between mobile radiation defects and pinning centers (for example, vacancy clusters), and show that the presence of pinning centers explains the unusual trajectories of migration of defects observed in in situ electron microscope experiments on ion-irradiated materials. ${ }^{4-6}$ 


\section{BROWNIAN MOTION OF INTERACTING NANODISLOCATION LOOPS: IN SITU ELECTRON MICROSCOPE OBSERVATIONS}

In situ electron microscope observations show two distinctly different modes of microstructural evolution, and two different types of defect dynamics, the occurrence of which depends on the type of particles irradiating the material. The ultrahigh-energy electron irradiation ${ }^{1-3}$ produces individual Frenkel pairs of vacancies and self-interstitial atoms, and the resulting supersaturation of defects gives rise to the nucleation and growth of small self-interstitial dislocation loops and vacancy clusters. Ion-beam irradiation ${ }^{4-6}$ generates collision cascades in which self-interstitial and vacancy defects form clusters as cascades cool down and resolidify. ${ }^{11,16,36}$

In the case of ultrahigh-energy electron irradiation, 99.998 wt \% pure bcc Fe (the metal most extensively studied by in situ microscopy so far) was used for making specimens. The impurity content characterizing the specimens is fully described in the supporting online material for Ref. 2. The specimens were rolled into 0.08-mm-thick sheets, which were preannealed at $1073 \mathrm{~K}$ for $2 \mathrm{~h}$ in a hydrogen atmosphere, and electrochemically polished. The orientation of the specimen surfaces was set close to (110) to minimize the image force acting on loops whose Burgers vectors $\mathbf{b}$, defining the directions in which the loops migrate, were $1 / 2$ [111] or $1 / 2[11 \overline{1}]$. High-energy electron irradiation was performed in an ultrahigh-voltage electron microscope H-3000 (Hitachi) operated at an acceleration voltage of $2000 \mathrm{kV}$. The dynamics of formation and migration of small dislocation loops was observed under electron irradiation. In another set of experiments, the dynamics of thermal Brownian motion of nanoscale dislocation loops initially produced by electron irradiation was observed using sample heating in an analytical H-800 (Hitachi) microscope operated at a relatively low acceleration voltage of $200 \mathrm{kV}$, at which no further radiation damage is produced. We note here that observing the migration of dislocation loops in an electron microscope inevitably gives rise to the electron beam itself affecting the observed mobility of the loops. ${ }^{37,38}$ This electron-beam effect does not significantly affect experimental observations described in this paper, making it possible to attribute the observed mobility of dislocation loops to thermal activation.

Specimen heating was performed following ultrahighenergy electron irradiation at temperatures ranging from 110 to $200 \mathrm{~K}$. The specimens were heated to temperatures in the range between 290 and $850 \mathrm{~K}$. Bright-field imaging was used for areas of the specimen where thickness varied between 100 to $300 \mathrm{~nm}$. The observation axis was approximately

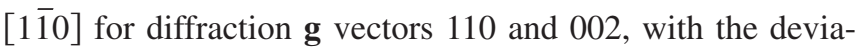
tion parameter from the exact Bragg condition $s$ ranging from 0.02 to $0.06 \mathrm{~nm}^{-1}$. Images were recorded using a silicon intensifier target camera with the time resolution of 1/30 s. It was possible to observe dislocation loops with diameters greater than a few nanometers.

In situ electron microscope observations show that the dynamics of motion of loops is visibly affected by elastic interactions between the loops. For example, Fig. 1 shows that three mobile prismatic dislocation loops formed in pure

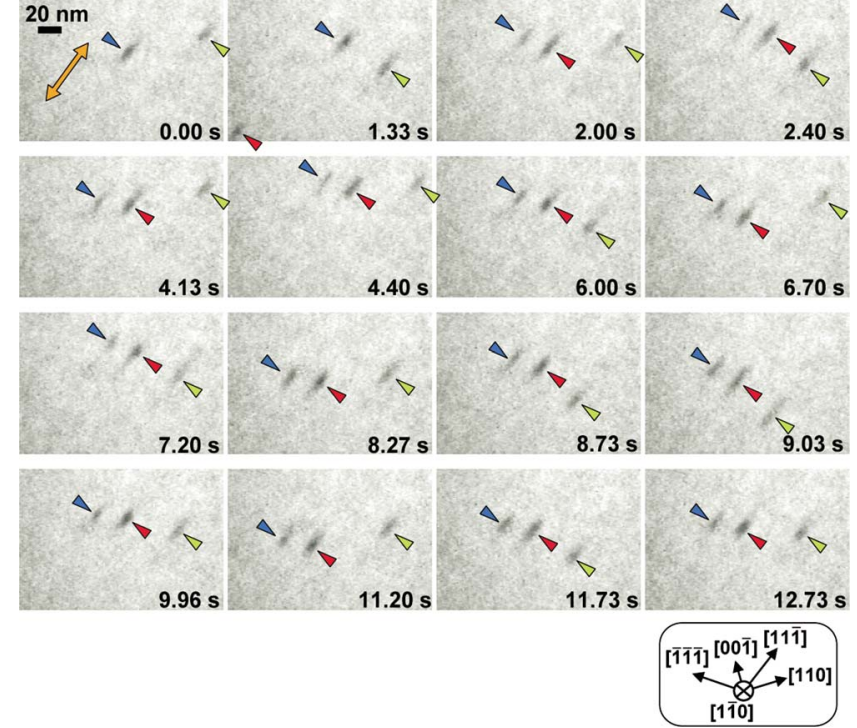

FIG. 1. (Color online) A sequence of in situ electron microscope snapshots recorded for $\mathbf{g}=002$ diffraction conditions at a $200 \mathrm{kV}$ accelerating voltage, and showing the evolution of an ensemble of three prismatic $a / 2[11 \overline{1}]$ dislocation loops formed in nominally pure iron by ultrahigh-voltage electron irradiation prior to the in situ experiment. The loops perform one-dimensional Brownian motion in the direction parallel to their Burgers vector at $T=673 \mathrm{~K}$. The loop diameters, from left to right, are $6,6.5$, and $6 \mathrm{~nm}$. The projected separation between the glide cylinders for the loops on the left (L) and in the center $(\mathrm{C})$ is $23 \mathrm{~nm}$, and the projected separation between the glide cylinders for the loops in the center $(\mathrm{C})$ and on the right $(R)$ is $41 \mathrm{~nm}$.

iron under ultrahigh-voltage electron irradiation, and initially separated by large distances, perform Brownian motion along their glide cylinders, and eventually approach each other closely enough so that the dynamics of loops becomes correlated due to elastic interaction between the loops. The loops eventually form a raft that migrates as a single entity, then grows and gradually coalesces into a single loop.

Other examples found by in situ electron microscope examination of specimens irradiated by ultrahigh-energy electrons show processes of capture of a mobile loop by the elastic field of another loop, followed by the coalescence of the loops. In general, the trend seen in in situ electron microscope observations of defects migrating in high-purity iron, and illustrated in Fig. 2, is that the motion of the defects is highly correlated, and the typical Brownian trajectories of defects follow each other on a $\sim 0.1 \mathrm{~s}$ time scale.

For ion-beam irradiation experiments, we used highpurity $\mathrm{Fe}$ (containing $\sim 1 \mathrm{ppm}$ carbon, $<5 \mathrm{ppm}$ nitrogen, $<10 \mathrm{ppm}$ silicon, and very small quantities of other impurities, which is not dissimilar to the impurity content of the specimens used for ultrahigh-energy electron irradiation experiments). The cold-rolled as-received material was annealed in vacuum at $1073 \mathrm{~K}$ for an hour followed by slow cooling. After this treatment all specimens had a simple ferritic microstructure with a low dislocation density. Thin foils were prepared by electropolishing and irradiated with 100 or $150 \mathrm{keV} \mathrm{Fe}^{+}$and $\mathrm{Xe}^{+}$ions at room temperature, and at 573 and $773 \mathrm{~K}$ in the Argonne IVEM-Tandem Facility. ${ }^{39}$ The 


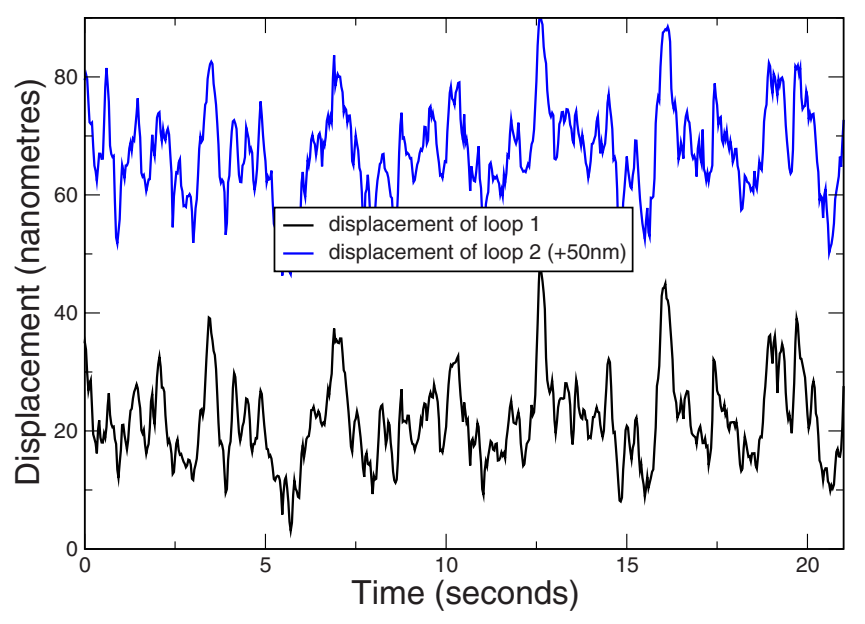

FIG. 2. (Color online) Trajectories of motion for two interacting $d=16 \mathrm{~nm}$ and $d=15 \mathrm{~nm}$ prismatic $a / 2\langle 111\rangle$ dislocation loops migrating in pure iron foil at $T=650 \mathrm{~K}$. The thickness of the foil is approximately $250 \mathrm{~nm}$ and the glide cylinders of the loops are 37 $\mathrm{nm}$ apart, as measured using stereo microscopy. The loops were formed by ultrahigh-energy electron irradiation followed by specimen annealing.

microscope was operated at $200 \mathrm{keV}$, below the threshold for knock-on radiation damage in Fe. Dynamic observations followed the evolution of damage over doses up to $13 \mathrm{dpa}$, according to a SRIM calculation with a displacement energy of $24 \mathrm{eV}$. Irradiations were paused from time to time to allow detailed characterization of microstructures using a number of diffraction-contrast techniques. ${ }^{4-6}$ Similar detailed characterization was performed at the end of the irradiation, after the specimens irradiated at elevated temperatures had cooled to room temperature.

The pattern of migration of nanodislocation loops in ionirradiated ultrahigh-purity (uhp) iron is surprisingly different from that of loops migrating in uhp iron irradiated with ultrahigh-energy electrons (see Fig. 2). This fact was highlighted in Ref. 40 (see Fig. 3 of Ref. 40, which shows how different are the observed and simulated trajectories of defect migration), and was noted in Refs. 4 and 6.

Figure 3 shows trajectories of migration for two nanodislocation loops in ion-irradiated iron. The trajectories exhibit a significant degree of correlation, similarly to the case of three migrating loops illustrated in Fig. 1. However, as opposed to the case of electron-irradiated iron, the trajectories of loops migrating in ion-irradiated iron do not look like the characteristic "fractal" Brownian trajectories shown in Fig. 2 and predicted by MD simulations. ${ }^{15,35}$ The trajectories showed in Fig. 3 demonstrate that the motion of loops consists of a series of relatively infrequent long-range "instantaneous" jumps, separated by extended intervals of time, during which the loops are pinned at certain points in the specimen and remain effectively immobile. Here we draw the attention of the reader again to the large difference between the time scales characterizing experimental observations ( $\sim 20 \mathrm{~s}$ for the examples shown in Figs. 2 and 3$)$ and MD simulations $\left(\sim 10^{-9} \mathrm{~s}\right.$ for the cases investigated in Refs. 15 and 35 ).

In subsequent sections of the paper, we show that the occurrence of correlated motion of loops, and the unusual

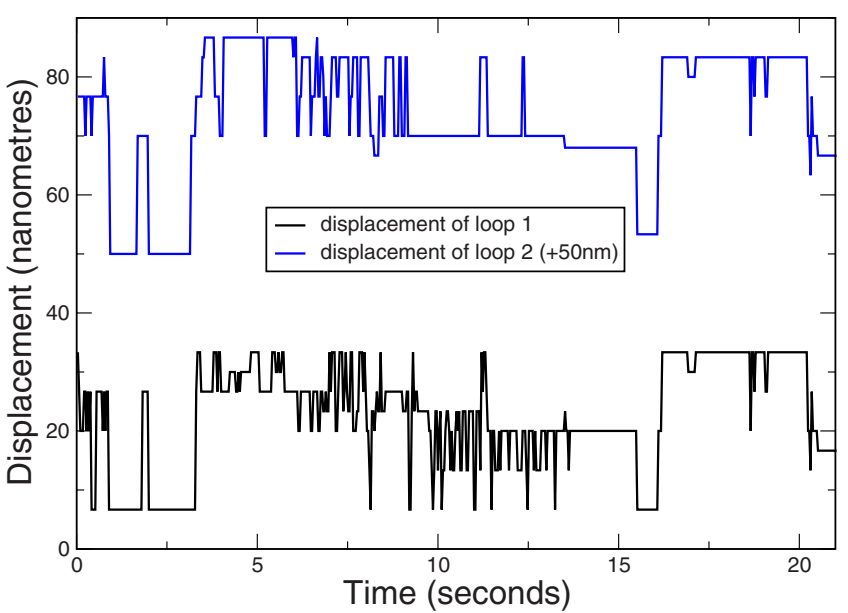

FIG. 3. (Color online) Experimentally observed trajectories of migration for two prismatic $\mathbf{b}=a / 2[111]$ dislocation loops, both of similar size $d \approx 4 \mathrm{~nm}$, performing thermally activated Brownian motion in pure iron after the specimen was irradiated with $150 \mathrm{keV}$ $\mathrm{Fe}^{+}$ions to a dose of $\sim 0.65 \mathrm{dpa}$. The projected distance between the glide cylinders of the loops is $12 \mathrm{~nm}$. The temperature of the specimen is $673 \mathrm{~K}$. Note the significant degree of correlation between the trajectories of motion of the loops shown in this figure.

shape of the trajectories of loops observed in experiments on ion-irradiated materials, can be explained if we assume that individual loops interact with other loops, and that they also interact with the "invisible" elements of microstructure, for example, small vacancy clusters formed in collision cascades generated by ion irradiation. ${ }^{41}$

We first investigate effects of migration of interacting loops using MD simulations, and then generalize the treatment to the case of Langevin dynamics of interacting defects. Using the latter method, we are able to match the time scales of experimental observations and simulations over intervals of time many orders of magnitude longer than those accessible to MD simulations. By assuming that loops interact via long-range elastic forces, we simulate the trajectories of correlated Brownian motion of the loops, and show that those also match experimental observations. Finally, using the isotropic elasticity expressions for the energy of interaction between the self-interstitial atom loops and vacancy clusters, we are able to simulate the unusual "pinned" Brownian trajectories of loops found experimentally in ionirradiated iron and shown in Fig. 3.

\section{A MOLECULAR DYNAMICS MODEL FOR INTERACTING NANODISLOCATION LOOPS}

We start our analysis with an MD investigation of how two small prismatic dislocation loops migrate if the glide cylinders of the loops are in close proximity to each other. Simulations were performed using the recent "magnetic" interatomic potential ${ }^{42}$ for $\alpha$-Fe. Two 61-SIA (self-interstitial atom) $\frac{1}{2}\langle 111\rangle$ dislocation loops were inserted into a regular $30 \times 20 \times 60$ cell $[$ in the $(x, y, z)=([1 \overline{1} 0],[11 \overline{2}],[111])$ coordinate system] containing 216000 atoms with periodic boundary conditions in all coordinate directions. These two 


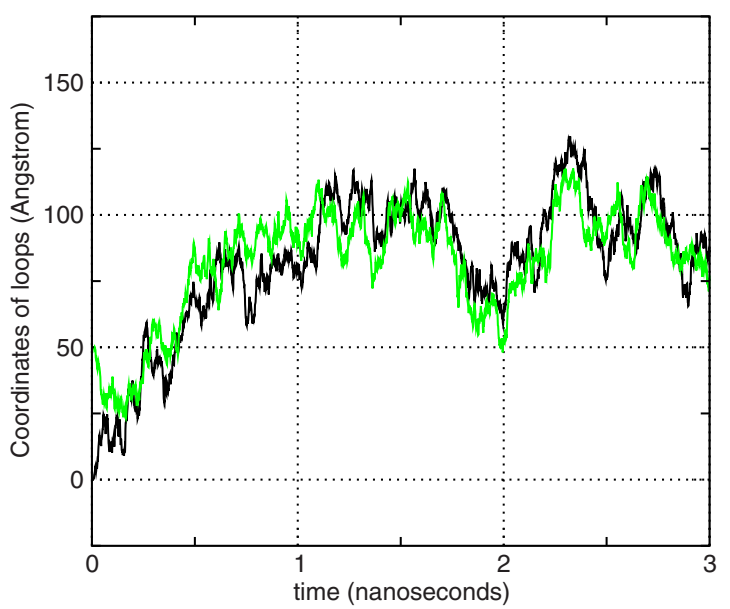

FIG. 4. (Color online) Trajectories of motion, simulated using molecular dynamics, for two interacting $\mathbf{b}=a / 2[111] 61$-atom $(d \approx 1.8 \mathrm{~nm})$ prismatic dislocation loops migrating in iron at $T=500 \mathrm{~K}$. The centers of loops are separated in the $[1 \overline{1} 0]$ direction by the distance $l \approx 40.5 \AA$.

$18.7 \AA$ diameter loops were inserted with their centers separated by $l \approx 40.5 \AA$ in the $[1 \overline{1} 0]$ direction but with the same

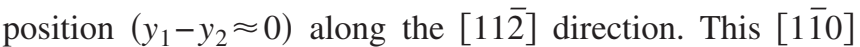
separation is comparable with the size of the loops themselves but is still large enough to ensure that there is no overlap between the core regions of the edge dislocations forming the loops so that all the interaction effects found in simulations are attributable to the long-range elastic fields of the loops. Note that this $\sim 4 \mathrm{~nm}$ interaction distance in $x$ $=[1 \overline{1} 0]$ is smaller than the separation between the loops through the $x$-direction periodic boundary $(\approx 8 \mathrm{~nm})$, which means that the "internal" $4 \mathrm{~nm}$ elastic interactions dominate. Each loop was given a random initial position along its respective $z=[111]$ glide cylinder.

After relaxing (quenching to $0 \mathrm{~K}$ ) the simulation cell, a sequence of $5 \mathrm{ps}$ finite-temperature simulations were performed at $100 \mathrm{~K}$ intervals to bring the system up to the 500 $\mathrm{K}$ temperature corresponding to the result presented here. The evolution of the two-loop system was simulated for a 3 ns interval at $500 \mathrm{~K}$, during which the positions of the loops were measured every $1 \mathrm{ps}$. The resulting one-dimensional [111] trajectories of the loops generated during this simulation are shown in Figs. 4 and 5. Following a method similar to that described in Refs. 34 and 35, we calculate separate diffusion coefficients $D$ for the motion of the loops, and find a value of approximately $5 \times 10^{9} \mathrm{~nm}^{2} \mathrm{~s}^{-1}$ for both loops.

There are two aspects of MD simulations described here that are difficult to relate to experimental observations (see Figs. 1 and 3). The difference between the time scales of MD simulations and experimental observations is on the order of $10^{9}$. Also, the diffusion coefficients for the loops derived from MD simulations are many orders of magnitude larger than those found experimentally. ${ }^{2}$ Hence, we do not attempt to directly compare MD simulations and experimental observations, and instead focus on the qualitative aspects of simulations that offer some insight into the origin of the effects observed experimentally.

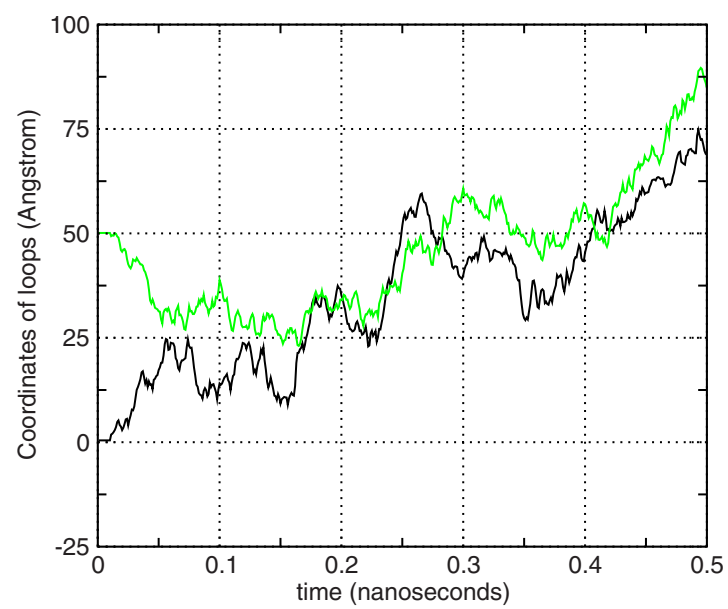

FIG. 5. (Color online) Trajectories of motion for interacting loops simulated using molecular dynamics and shown in Fig. 4 but plotted for a shorter interval of time. Initially the loops are $5 \mathrm{~nm}$ apart in the direction of their Burgers vector. Elastic interaction brings the loops together at $t \approx 0.17 \mathrm{~ns}$. After this moment, Brownian motion of the two loops becomes strongly correlated, the loops form a raft and migrate almost as a single entity.

The most notable feature seen in the simulations is the correlated nature of the migration of the loops. Figures 4 and 5 show that although initially the loops were separated, in the direction parallel to their Burgers vector (which is the direction of one-dimensional Brownian motion of the loops), by a distance of approximately $5 \mathrm{~nm}$, after just $170 \mathrm{ps}$ the random Brownian motion of the loops became strongly correlated (see Fig. 5), and the trajectories of the loops during the rest of the time interval spanned by the simulation closely followed each other. The strongly correlated nature of Brownian motion of the loops found in MD simulations may appear exaggerated in comparison with experimental observations, where loops would occasionally drift apart and separate. However we note that the distance between the glide cylinders set up in MD simulations is almost an order of magnitude smaller in comparison with the distances between the glide cylinders for the loops seen in Fig. 1, and hence the strength of elastic interaction between the loops in the MD simulations is much larger than in observations illustrated in Fig. 1.

The main conclusion that we derive from these simulations, which illustrate the effect of interaction between the loops on their Brownian motion, is the emergence of a collective mode of motion, where trajectories of migration of loops, while exhibiting a considerable degree of randomness, follow each other over an extended period of time. We now show how this conclusion can be generalized and extended to the case of many interacting loops and much longer time scales through the use of the Langevin treatment of dynamics of loops.

\section{RANDOM THERMAL FORCE ACTING ON A DISLOCATION LOOP}

The fact that migration of individual self-interstitial crowdion defects and small dislocation loops could be rational- 


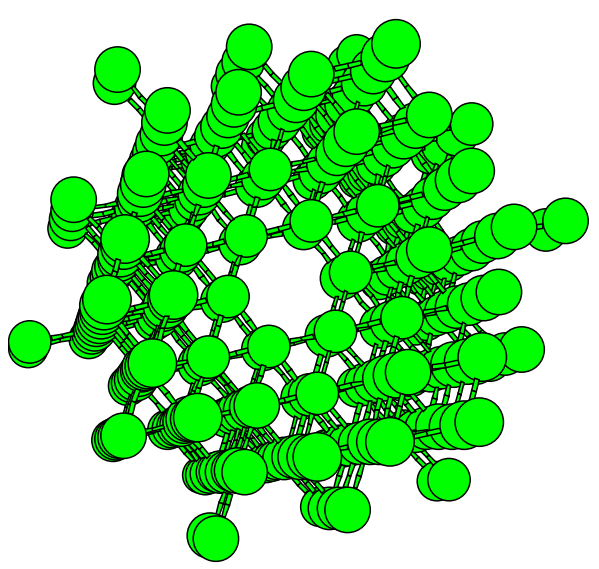

FIG. 6. (Color online) The equilibrium structure of a small dislocation loop formed by the agglomeration of 37 self-interstitial atoms on a (111) habit plane in bcc iron (Refs. 41 and 45). The Burgers vector of the loop is $\mathbf{b}=a / 2[111]$, where $a$ is the lattice constant. Only those atoms are shown, the potential energy of which exceeds by $0.05 \mathrm{eV}$ the potential energy of an atom in a perfect lattice. The viewing direction is slightly off the $\langle 111\rangle$ axis, which is parallel to the Burgers vector of the loop.

ized using a Langevin-equation-based treatment was discussed in Refs. 34, 35, 37, and 43. In this section we prove, using the multistring Frenkel-Kontorova model, ${ }^{44}$ that the center of mass of a loop moves under the action of random thermal force resulting from thermal vibrations of atoms at the perimeter of the loop, or, in other words, that a small dislocation loop can be treated as a particle, the coordinate of which satisfies the Langevin equation of motion. Figure 6 shows the structure of a 37-self-interstitial-atom dislocation loop formed in bcc iron by the agglomeration of selfinterstitial atoms on the (111) habit plane. The Burgers vector of the loop, the direction of which is parallel to the atomic strings shown in Fig. 6, is $\mathbf{b}=a / 2$ [111]. Each atomic string, depending on its position in the loop, is described by the profile of atomic displacements in the direction parallel to the Burgers vector $\mathcal{U}_{\mathbf{j}}\left[n-\mathcal{Z}_{\mathbf{j}}(t) / b\right]$, where $n$ is the index of an atom in a string, and $\mathbf{j}$ is the two-dimensional index of a string in the plane normal to the plane of the loop. ${ }^{34,44} \mathcal{Z}_{\mathbf{j}}(t)$ is the time-dependent position of the center of the $\mathbf{j t h}$ atomic displacement profile, which in the case of a single selfinterstitial atom defect (a crowdion) can be identified with the position of the defect in the crystal lattice.

By following the method described in Refs. 34, 35, and 37 , we find a set of coupled equations of motion for the coordinates of centers of displacement profiles associated with atomic strings running in a $\langle 111\rangle$-type direction,

$$
\begin{aligned}
m \frac{d^{2} \mathcal{Z}_{\mathbf{j}}(t)}{d t^{2}}= & 4 m \omega^{2} \sum_{n, \mathbf{h}}\left[\Phi_{n, \mathbf{j}}(t)-\Phi_{n, \mathbf{j}+\mathbf{h}}(t)\right] \\
& \times \sin ^{2}\left\{\frac { \pi } { a } \left\{\mathcal{U}_{\mathbf{j}}\left[n-\mathcal{Z}_{\mathbf{j}}(t) / a\right]\right.\right. \\
& \left.\left.-\mathcal{U}_{\mathbf{j}+\mathbf{h}}\left[n-\mathcal{Z}_{\mathbf{j}+\mathbf{h}}(t) / a\right]\right\}\right\} .
\end{aligned}
$$

Here $\Phi_{\mathbf{j}, n}(t)$ denotes the field of random (thermal) displacements of atoms in the strings, as opposed to the regular field of time-dependent coordinate $\mathcal{Z}_{\mathbf{j}}(t)$, which describes migration of the center of an atomic displacement profile as a whole. The number of equations in the set (1) equals the number of interstitial atoms in the loop. The strings forming the lattice surrounding the loop are assumed to be unaffected by the elastic field of the loop [in other words, for the strings surrounding the loop, $\left.\mathcal{Z}_{\mathbf{j}}(t)=0\right]$. However, atoms in the lattice surrounding the loop still undergo random thermal motion and interact with atoms in the strings on the perimeter of the loop.

The position of the center of mass of the loop is given by

$$
\mathcal{Z}(t)=\frac{1}{N_{s}} \sum_{\mathbf{j}} \mathcal{Z}_{\mathbf{j}}(t),
$$

where $N_{s}$ is the number of atomic strings in the loop (for example, for the case shown in Fig. 6, $N_{s}=37$ ). Performing the summation over all the strings $\mathbf{j}$ forming the loop, and noting that random forces acting on the neighboring strings enter the sum with opposite signs, and hence fully compensate each other, we find that the equation of motion for the center of mass of a loop only contains terms describing thermal forces acting on strings situated at the perimeter of the loop,

$$
\begin{aligned}
m \frac{d^{2} \mathcal{Z}(t)}{d t^{2}}= & 4 m \omega^{2} \sum_{n, \mathbf{P}} \sum_{\mathbf{h}^{\prime}}\left[\Phi_{n, \mathbf{P}}(t)-\Phi_{n, \mathbf{P}+\mathbf{h}^{\prime}}(t)\right] \\
& \times \sin ^{2}\left\{\frac{\pi}{a} \mathcal{U}_{\mathbf{P}}\left[n-\mathcal{Z}_{\mathbf{P}}(t) / a\right]\right\} .
\end{aligned}
$$

Here the summation over $\mathbf{P}$ is performed over strings situated at the perimeter of the loop, where fluctuating phonon forces are not compensated, and summation over $\mathbf{h}^{\prime}$ is performed over strings in the lattice around the dislocation loop. The right-hand side of Eq. (3) equals the projection (on the direction of the Burgers vector of the loop) of the total timedependent random phonon force $f(t)$ acting on the loop. It is known that a random force acting on a particle results in the particle performing Brownian motion. At the same time, according to the fluctuation-dissipation theorem, the presence of randomly fluctuating forces give rise to thermal friction. ${ }^{46}$

Applying the Einstein model for thermal vibrations of atoms to Eq. (3), ${ }^{34}$ we find that the correlation function of random force acting on a loop is proportional to the length $L$ of the perimeter of the loop,

$$
\overline{f(t) f\left(t^{\prime}\right)}=f^{2} \delta\left(t-t^{\prime}\right) \sim L \delta\left(t-t^{\prime}\right) .
$$

Using the fluctuation-dissipation theorem, we find the coefficient of thermal friction for the loop, ${ }^{34}$

$$
\gamma=\frac{f^{2}}{2 k_{B} T} .
$$

The diffusion coefficient $D$ is related to the friction coefficient $\gamma$ via $D=k_{B} T / \gamma$. This equation shows that the diffusion coefficient for a loop treated as a function of its size varies approximately as 


$$
D \sim L^{-1},
$$

where $L$ is the perimeter of the loop. In what follows, we use this relation to evaluate the diffusion coefficients for loops of various sizes by extrapolating the experimentally measured values for diffusion coefficients found for a loop of a certain size. $^{2,3}$ We note that the dependence of the diffusion coefficient for the center of mass of the loop on the length of the perimeter of the loop predicted by the Frenkel-Kontorova model described above agrees well with results of MD simulations, ${ }^{15}$ where it was found that the diffusion coefficient depends on the total number of self-interstitial atoms forming the loop $N_{s}$ as $D \sim N_{s}^{-0.64}$, which is close to $D$ $\sim N_{s}^{-1 / 2}$ expected from Eq. (6) in the limit of large loop size. Experimental observations are also broadly in agreement with the above $D \sim L^{-1}$ law. ${ }^{2,3}$

We note that even in a pure material the diffusion coefficient for a loop is strongly influenced by the presence of impurities, which form a mobile "cloud" around the loop. The presence of this mobile atmosphere of impurities renormalizes the mobility of a loop, strongly increasing the effective activation energy for migration in comparison with the estimates derived from MD simulations., ${ }^{2,3}$

\section{A LANGEVIN DYNAMICS MODEL FOR BROWNIAN MOTION OF INTERACTING NANODISLOCATION LOOPS}

In the previous section, we showed that the center of mass of a dislocation loop moves under the action of an effective random force associated with the thermal motion of atoms in the material. This effective fluctuating thermal force induces stochastic Brownian motion of loops observed in in situ electron microscope experiments ${ }^{1-6}$ and in MD simulations. ${ }^{10-15}$ Similarly, two nanodislocation loops formed in close proximity of each other, and interacting via long-range elastic forces, perform correlated Brownian motion, as illustrated in Figs. 4 and 5.

The diffusion equation for a system of $N$ interacting particles has the form ${ }^{47}$

$$
\frac{\partial P}{\partial t}=\sum_{i=1}^{N} D_{i}^{\alpha \beta}\left(\frac{\partial^{2} P}{\partial r_{i}^{\alpha} \partial r_{i}^{\beta}}+\frac{1}{k_{B} T}\left[\frac{\partial U}{\partial r_{i}^{\alpha}}\right]\left[\frac{\partial P}{\partial r_{i}^{\beta}}\right]\right),
$$

where $\alpha, \beta=(x, y, z), P=P\left(\mathbf{r}_{1}, \mathbf{r}_{2}, \ldots, \mathbf{r}_{N}\right)$ is a $N$-dimensional probability distribution function, $D_{i}^{\alpha \beta}$ is the diffusion matrix for particle $i$, and $T$ is the absolute temperature. The function $U=U\left(\mathbf{r}_{1}, \mathbf{r}_{2}, \ldots, \mathbf{r}_{N}\right)$ describes the interaction between the particles, and the gradient of this function $F_{i}^{\alpha}=-\partial U / \partial r_{i}^{\alpha}$ gives the $\alpha$ component of the force acting on particle $i$.

In the limit of strongly anisotropic one-dimensional diffusion, describing random thermal glide of glissile nanodislocation loops, Eq. (7) can be simplified as

$$
\frac{\partial P}{\partial t}=\sum_{i=1}^{N} D_{i}\left(\frac{\partial^{2} P}{\partial z_{i}^{2}}+\frac{1}{k_{B} T}\left[\frac{\partial U}{\partial z_{i}}\right]\left[\frac{\partial P}{\partial z_{i}}\right]\right) .
$$

Here $D_{i}$ is the diffusion coefficient for loop $i$, and $z_{i}$ is the projection of the center of mass of the loop on the direction of its Brownian motion. We assume that all the loops have the same Burgers vector, which defines the orientation of the glide cylinders and the direction of Brownian motion for the loops.

Equation (8) describes a relatively simple model for an ensemble of interacting Brownian particles, which is even simpler than models considered previously in the context of Brownian dynamics of particles suspended in a fluid and interacting via hydrodynamic forces. ${ }^{48,49}$ In principle, the evolution of this model could be investigated using kinetic Monte Carlo simulations, following the method developed by Hudson et al. ${ }^{27}$ who introduced hopping probabilities biased by long-range elastic fields. However, since implementing this approach requires synchronizing the Monte Carlo events, which presents a potentially difficult issue for parallelizing the kinetic Monte Carlo algorithm, ${ }^{31}$ here we adopt a different strategy and instead focus on a set of differential equations for the coordinates of the loops. To achieve this, we map Eq. (8) onto a set of $N$ coupled overdamped Langevin equations of the form

$$
\frac{d z_{i}}{d t}=-\frac{D_{i}}{k_{B} T} \frac{\partial U}{\partial z_{i}}+\sqrt{2 D_{i}} \xi_{i}(t), \quad i=1,2, \ldots, N,
$$

where $\xi_{i}(t)$ are random variables satisfying the condition $\overline{\xi_{i}(t) \xi_{i}\left(t^{\prime}\right)}=\delta\left(t-t^{\prime}\right)$ and $U\left(z_{1}, z_{2}, \ldots, z_{N}\right)$ is the energy of interaction between the loops. The energy of interaction between the loops is, of course, also a function of the position of the loops in the $(x, y)$ plane. However, since the loops do not change their $x$ and $y$ coordinates, we omit explicit reference to these degrees of freedom.

The mathematical equivalence between the overdamped Langevin Eq. (9) and the many-body diffusion Eq. (8) exists independently of the microscopic nature of processes responsible for the diffusion of loops ${ }^{50,51}$ since the only parameters entering both equations are the diffusion coefficients $D_{i}$ for the particles. Hence statistical simulations of trajectories of loops by means of the Langevin Eq. (9) are fully equivalent to finding a time-dependent ensemble-averaged solution of a many-body diffusion Eq. (8). The significant advantage offered by the Langevin equation simulation approach is that by solving these equations we could follow the trajectories of the defects, and compare them with the trajectories observed by in situ electron microscopy. This contrasts with the solutions of the many-body diffusion equation, which describe the evolving microstructure in the statistical ensemble sense. For example, solutions of a many-body diffusion equations cannot be directly compared with the trajectories of migrating loops observed in in situ experiments.

\section{A. Interaction between an interstitial loop and a vacancy cluster}

To investigate the role played by the long-range interaction between an interstitial loop and an immobile pinning center (a vacancy cluster or an impurity), we use expressions derived from the theory of elasticity. In the isotropic approximation of the theory of elasticity, the energy of interaction between a loop and a spherical inclusion is ${ }^{52}$ 


$$
\begin{aligned}
U(z)= & \frac{\Delta V}{3 \pi} b \mu \frac{1+\nu}{1-\nu} \frac{1}{\sqrt{z^{2}+(R+r)^{2}}} \\
& \times\left[\frac{R^{2}-r^{2}-z^{2}}{(R-r)^{2}+z^{2}} E\left(\sqrt{\frac{4 r R}{z^{2}+(R+r)^{2}}}\right)\right. \\
& \left.+K\left(\sqrt{\frac{4 r R}{z^{2}+(R+r)^{2}}}\right)\right] .
\end{aligned}
$$

Here $R$ is the radius of the loop, functions $K(k)$ and $E(k)$ are, respectively, the complete elliptic integrals of the first and second kind, and $r$ and $z$ are the cylindrical coordinates, the origin of which corresponds to the center of the dislocation loop. $\Delta V$ is the relaxation volume (a quantity which has negative sign in the case of a vacancy or a vacancy cluster), $\mathbf{b}$ is the Burgers vector of the loop, $b=|\mathbf{b}|$, and $\mu$ and $\nu$ are the shear modulus of the material and the Poisson ratio.

Although, in principle, Eq. (10) applies equally to an interstitial and a vacancy loop (where in the latter case, the energy of interaction has the opposite sign), the fact that a vacancy loop is a metastable configuration that has higher energy than a spherical vacancy cluster (a void) ${ }^{45}$ leads to interstitial loops dominating the observed microstructure of an irradiated material. ${ }^{6}$ In what follows we only consider the case of an interstitial dislocation loop that is attracted to a vacancy cluster situated at the axis of the glide cylinder for the loop. In the limit of large separation between the loop and the inclusion $r \gg R, z \gg R$, functions $K(k)$ and $E(k)$ can be expanded in the Taylor series as (note that the definition of these functions differs from that given in Abramovitz and Stegun's handbook ${ }^{53}$ ),

$$
\begin{aligned}
& K(k) \approx \frac{\pi}{2}\left(1+\frac{k^{2}}{4}+\frac{9 k^{4}}{64}-\cdots\right), \\
& E(k) \approx \frac{\pi}{2}\left(1-\frac{k^{2}}{4}-\frac{3 k^{4}}{64}-\cdots\right),
\end{aligned}
$$

resulting in the far field form of Eq. (10),

$$
U(z)=-\frac{\Delta V}{6} b \mu \frac{1+\nu}{1-\nu} R^{2} \frac{1-3 \cos ^{2} \theta}{l^{3}},
$$

where $z / l=\cos \theta, r / l=\sin \theta$, and $l=\sqrt{r^{2}+z^{2}}$.

Equation (10) acquires a particularly simple form for $r$ $=0$, corresponding to the case where the inclusion is situated at the axis of the glide cylinder for the loop,

$$
U(z)=\frac{\Delta V}{3 \pi} \mu \frac{1+\nu}{1-\nu} \frac{\pi R^{2} b}{\left(z^{2}+R^{2}\right)^{3 / 2}} .
$$

This equation shows that the range of the force field describing elastic interaction between a dislocation loop and an inclusion is of the same order of magnitude as the loop radius $R$.

Figures 7 and 8 show examples of the potential-energy curves describing elastic interactions between a dislocation loop and a single vacancy or a vacancy cluster. The discrete points are evaluated using atomistic total-energy conjugate gradient minimization for a 313-self-interstitial atom loop interacting with a single vacancy situated at the center of the

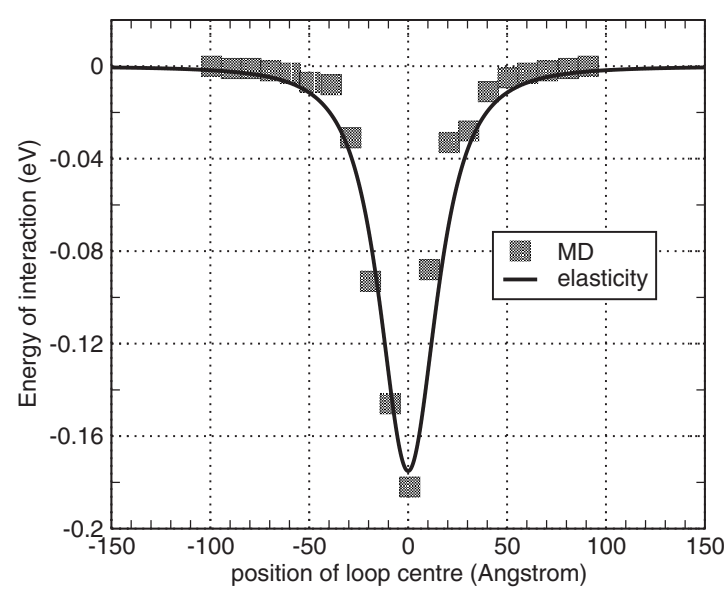

FIG. 7. Energy of interaction between a 313-atom ( $R$ $=2.2 \mathrm{~nm})$ self-interstitial $\mathbf{b}=a / 2\langle 111\rangle$ dislocation loop and a vacancy shown as a function of separation $z$ between the center of the loop and the vacancy. The vacancy is situated at the axis of the glide cylinder $r=0$. The solid line follows Eq. (10) for $\Delta V$ $=-5 \AA^{3}, \nu=0.29$, and $\mu=82 \times 10^{9} \mathrm{~Pa}$.

glide cylinder, or a 10-vacancy cluster situated at the center of the glide cylinder. The solid curves were calculated using Eq. (10) and isotropic elastic parameters for pure iron. The effective formation volumes $\Delta V$ for a single vacancy and a vacancy cluster were chosen to fit the MD simulation data. These values are broadly in agreement with the literature data on formation volumes of vacancy defects, ${ }^{54,55}$ which themselves are subject to considerable uncertainty and fluctuations. We see that, depending on the formation volume of a vacancy defect, the magnitude of interaction between a self-interstitial loop and a vacancy defect $U(z)$ varies between 0.2 and $1 \mathrm{eV}$. The width of the potential wells shown in Figs. 7 and 8 are comparable with the diameter of the dislocation loop.

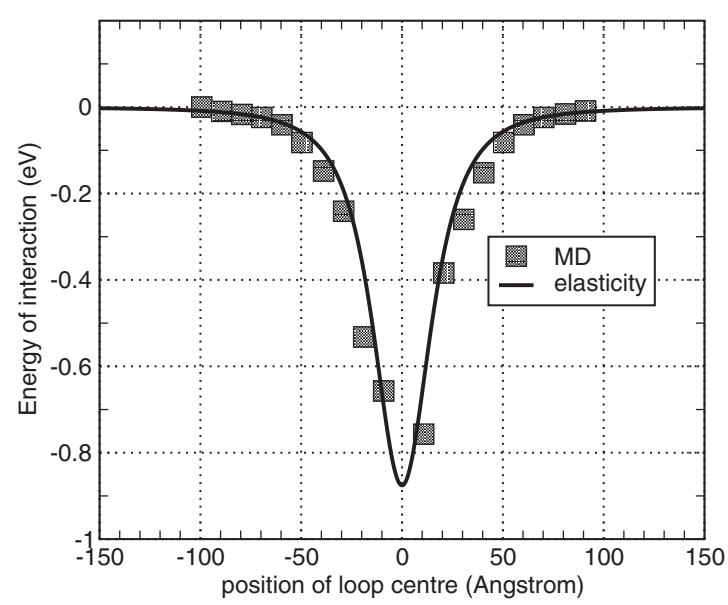

FIG. 8. Energy of interaction between a 313-atom $(R$ $=2.2 \mathrm{~nm})$ self-interstitial $\mathbf{b}=a / 2\langle 111\rangle$ dislocation loop and a 10 vacancy cluster shown as a function of separation $z$ between the center of the loop and the cluster. The vacancy cluster is situated at the axis of the glide cylinder $r=0$. The solid line follows Eq. (10) for $\Delta V=-25 \AA^{3}, \nu=0.29$, and $\mu=82 \times 10^{9} \mathrm{~Pa}$. 


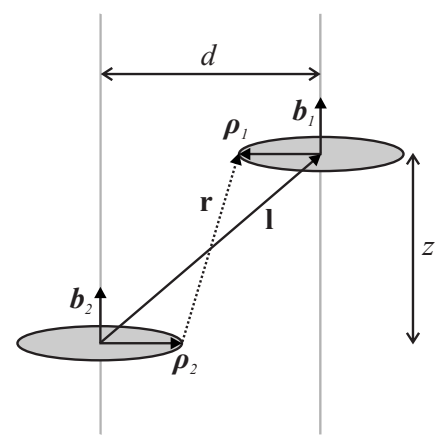

FIG. 9. Diagram illustrating the notations used in Eqs. (14) and (19).

\section{B. Interaction between two dislocation loops}

The energy of interaction between two circular dislocation loops with parallel Burgers vectors $\mathbf{b}_{1}$ and $\mathbf{b}_{2}$ is given by [see Eqs. (4)-(23) of Hirth and Lothe $\left.{ }^{56}\right]$

$$
\begin{aligned}
U= & \frac{\mu b_{1} b_{2}}{4 \pi(1-\nu)} \int_{0}^{2 \pi} d \phi_{1} \int_{0}^{2 \pi} d \phi_{2}\left\{\frac{\left[\boldsymbol{\rho}\left(\phi_{1}\right) \cdot \boldsymbol{\rho}\left(\phi_{2}\right)\right]}{r}\right. \\
& \left.-\frac{\left[\mathbf{r} \cdot \boldsymbol{\rho}\left(\phi_{1}\right)\right]\left[\mathbf{r} \cdot \boldsymbol{\rho}\left(\phi_{2}\right)\right]}{r^{3}}\right\} .
\end{aligned}
$$

Here $\mathbf{r}=\mathbf{l}+\boldsymbol{\rho}\left(\phi_{1}\right)-\boldsymbol{\rho}\left(\phi_{2}\right)$ is a vector connecting points situated on the perimeter of the two loops, as shown in Fig. 9, and $\boldsymbol{\rho}\left(\phi_{1}\right)$ and $\boldsymbol{\rho}\left(\phi_{2}\right)$ are two radial vectors in the habit planes of the loops.

In the far field region, where $\left|\boldsymbol{\rho}\left(\phi_{1}\right)-\boldsymbol{\rho}\left(\phi_{2}\right)\right| \ll|\mathbf{I}|$, we find

$$
\frac{1}{r^{3}}=\frac{1}{|\mathbf{l}+\boldsymbol{\rho}|^{3}}=\frac{1}{l^{3}}-\frac{3(\boldsymbol{\rho} \cdot \mathbf{l})}{l^{5}}-\frac{3 \boldsymbol{\rho}^{2}}{2 l^{5}}+\frac{15(\boldsymbol{\rho} \cdot \mathbf{l})^{2}}{2 l^{7}}+\cdots,
$$

where $\boldsymbol{\rho}=\boldsymbol{\rho}\left(\phi_{1}\right)-\boldsymbol{\rho}\left(\phi_{2}\right)$, and the equation for the energy acquires the form

$$
\begin{aligned}
U= & \frac{\mu b_{1} b_{2}}{4 \pi(1-\nu)} \int_{0}^{2 \pi} d \phi_{1} \int_{0}^{2 \pi} d \phi_{2}\left[\left(\mathbf{l}^{2}+2 \mathbf{l} \cdot \boldsymbol{\rho}+\boldsymbol{\rho}^{2}\right)\left(\boldsymbol{\rho}_{1} \cdot \boldsymbol{\rho}_{2}\right)\right. \\
& \left.-\left([\mathbf{l}+\boldsymbol{\rho}] \cdot \boldsymbol{\rho}_{1}\right)\left([\mathbf{l}+\boldsymbol{\rho}] \cdot \boldsymbol{\rho}_{2}\right)\right]\left[\frac{1}{l^{3}}-\frac{3(\boldsymbol{\rho} \cdot \mathbf{l})}{l^{5}}-\frac{3 \boldsymbol{\rho}^{2}}{2 l^{5}}\right. \\
& \left.+\frac{15(\boldsymbol{\rho} \cdot \mathbf{l})^{2}}{2 l^{7}}+\cdots\right] .
\end{aligned}
$$

Noting that

$$
\begin{gathered}
\int_{0}^{2 \pi} d \phi_{1} \int_{0}^{2 \pi} d \phi_{2}\left(\boldsymbol{\rho}_{1} \cdot \boldsymbol{\rho}_{2}\right)\left(\boldsymbol{\rho}_{1} \cdot \mathbf{l}\right)\left(\boldsymbol{\rho}_{2} \cdot \mathbf{l}\right)=A_{1} A_{2} l^{2} \sin ^{2} \theta \\
\int_{0}^{2 \pi} d \phi_{1} \int_{0}^{2 \pi} d \phi_{2}\left(\boldsymbol{\rho}_{1} \cdot \mathbf{l}\right)^{2}\left(\boldsymbol{\rho}_{2} \cdot \mathbf{l}\right)^{2}=A_{1} A_{2} l^{2} \sin ^{4} \theta
\end{gathered}
$$

$$
\int_{0}^{2 \pi} d \phi_{1} \int_{0}^{2 \pi} d \phi_{2}\left(\boldsymbol{\rho}_{1} \cdot \boldsymbol{\rho}_{2}\right)^{2}=2 A_{1} A_{2}
$$

where $A_{1}=\pi \rho_{1}^{2}$ and $A_{2}=\pi \rho_{2}^{2}$ are the respective areas of the loops, we find

$$
\begin{aligned}
U & =\frac{\mu b_{1} b_{2}}{4 \pi(1-\nu)} \frac{A_{1} A_{2}}{l^{3}}\left(8-24 \sin ^{2} \theta+15 \sin ^{4} \theta\right) \\
& =-\frac{\mu b_{1} b_{2}}{4 \pi(1-\nu)} \frac{A_{1} A_{2}}{l^{3}}\left(1+6 \cos ^{2} \theta-15 \cos ^{4} \theta\right) .
\end{aligned}
$$

The expression for the energy of interaction between two circular loops [Eq. (18)] was derived by Foreman and Eshelby ${ }^{57}$ using the isotropic elasticity approximation, and reported in the literature by Barnes. ${ }^{58}$ The form of this equation is similar to that of Eq. (12). In both cases, the energy varies as a function of distance between the defects as $U$ $\sim l^{-3}$. We also see that the product of the Burgers vector and the area of the loop plays the part of the effective formation volume. Unsurprisingly, the characteristic scale of interaction energies (a fraction of an electron volt) is similar in both cases. For practical calculations, it is convenient to express formula (18) in terms of two variables, the distance $d$ between the glide cylinders of the loops and the distance $z$ between the habit planes of the loops. In terms of these variables, Eq. (18) can be written in the form

$U(z, d)=-\frac{\mu b_{1} b_{2}}{4 \pi(1-\nu)} \frac{A_{1} A_{2}}{\left(z^{2}+d^{2}\right)^{3 / 2}}\left[1+\frac{6 z^{2}}{\left(z^{2}+d^{2}\right)}-\frac{15 z^{4}}{\left(z^{2}+d^{2}\right)^{2}}\right]$.

Our analysis of experimental data on the statistics of migration of dislocation loops suggests that the Foreman-Eshelby expression (18) overestimates, at least for the case of the $\mathbf{b}$ $=a / 2\langle 111\rangle$ loops considered here, the height of potential barriers for reaction between the loops. The fact that Eqs. (14) and (18) for the energy of interaction between the loops may not be accurate is not surprising since, for example, it is known that even at room temperature, iron is elastically anisotropic. $^{7-9}$ Hence the isotropic elasticity formulas (14) and (18) should be treated as estimates for the strength of interaction as well as for the functional form for the law of interaction between the loops. The use of the isotropic elasticity approximation (where the energy of interaction between the loops depends only on the relative orientation of the Burgers vectors of the loops and the relative positions of the loops, and is independent of the orientation of the Burgers vector of the loop relative to the crystal lattice) has the advantage that the functional form for the energy of interaction between the loops is sufficiently simple, to enable fast evaluation of terms in the right-hand side of Eq. (9). The elastic anisotropy approximation does not change the general functional form for the energy of interaction between the loops, which at large distances between the loops varies as (distance $)^{-3}$ but it introduces an element of complexity in the angular dependence of the energy of interaction, as it was shown for the full anisotropic elasticity case by Willis. ${ }^{59}$ 


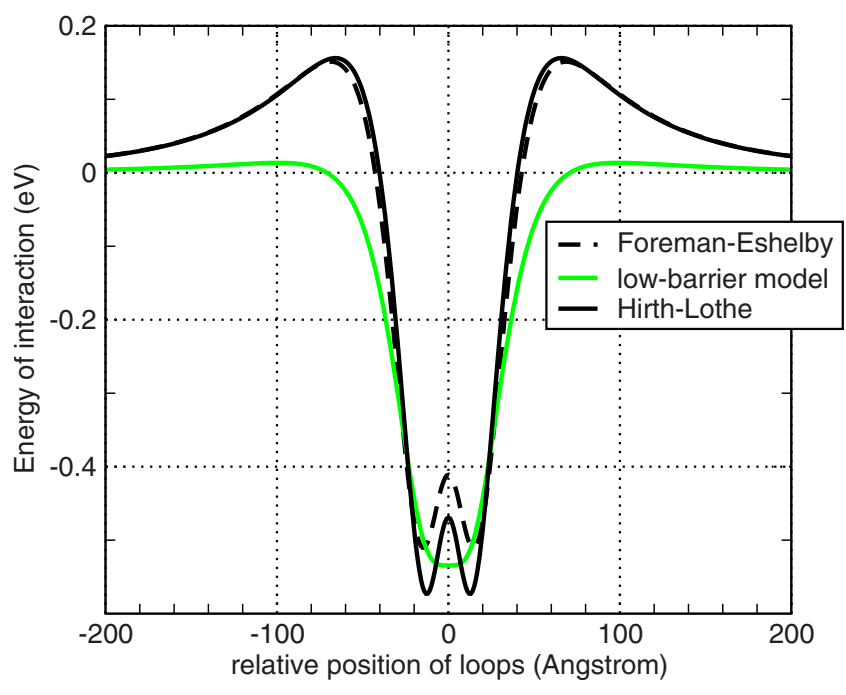

FIG. 10. (Color online) Energy of interaction between two circular $(R=9.4 \AA)$ self-interstitial $\mathbf{b}=a / 2\langle 111\rangle$ dislocation loops in iron evaluated using the "exact" isotropic elasticity expression (14), the Foreman-Eshelby expression (18), and the low-barrier model (20), where the curve describing interaction between the loops as a function $z$ exhibits low potential barriers for reaction between the loops. The elasticity calculations were performed assuming $\nu$ $=0.29$ and $\mu=82 \times 10^{9} \mathrm{~Pa}$.

In this study, together with the original Foreman-Eshelby expression (18), we also use an alternative form, motivated by experiment, for the energy of interaction (the "low-barrier model"), which we derive from Eq. (18) by modifying some of its numerical coefficients,

$$
\begin{aligned}
U(z, d)= & -\frac{\mu b_{1} b_{2}}{4 \pi(1-\nu)} \frac{A_{1} A_{2}}{\left(z^{2}+d^{2}\right)^{3 / 2}} \frac{13}{10} \\
& \times\left[1+\frac{1}{4} \frac{6 z^{2}}{\left(z^{2}+d^{2}\right)}-\frac{1}{4} \frac{15 z^{4}}{\left(z^{2}+d^{2}\right)^{2}}\right] .
\end{aligned}
$$

A curve calculated using Eq. (20) is shown in Fig. 10 together with the curves evaluated using Eqs. (14) and (18). Equation (20), where the energy of interaction between the loops is independent of the orientation of their Burgers vectors with respect to the crystal lattice, still refers to the isotropic elasticity approximation.

We note that the inclusion of anisotropic elasticity effects does not alter the general functional form of the laws describing interaction between the defects. Since in this work, we focus on the generic new features of Brownian motion of defects associated with the fact that defects interact, we do not specifically investigate the role played by the anisotropic elasticity effects.

In the next two sections of the paper, we apply the Langevin dynamics method to the treatment of migration of two and three interacting loops, and also investigate Brownian motion of an individual loop interacting with statistically distributed vacancy clusters.

\section{CORRELATED BROWNIAN MOTION OF NANODISLOCATION LOOPS}

In this section, we focus attention on solving Eq. (9) and on investigating the part played by the interaction between migrating nanodislocation loops. We find that the interaction between migrating loops, and between loops and vacancy clusters, acting as pinning centers for the loops, gives rise to striking deviations in the statistics of motion of loops from the statistics of "free" Brownian motion investigated in the past by MD simulations ${ }^{10-15}$ and analyzed using interactionfree Langevin dynamics equations. ${ }^{34,35,37,43}$ Also, the Langevin dynamics simulations described below use the effective values of diffusion coefficients, derived from experimental observations for freely migrating loops, which take into account the presence of intrinsic impurities.

We start by considering the case of two interacting $\mathbf{b}$ $=(a / 2)\langle 111\rangle$ loops of diameters $d_{1}=16 \mathrm{~nm}$ and $d_{2}=15 \mathrm{~nm}$ migrating in nominally pure iron at $T_{0}=650 \mathrm{~K}$. The diffusion coefficients for the loops, derived from the values measured for a single loop, and scaled using the inverse perimeter length law [Eq. (6)] are $D_{1}=296 \mathrm{~nm}^{2} \mathrm{~s}^{-1}$ and $D_{2}$ $=315 \mathrm{~nm}^{2} \mathrm{~s}^{-1}$. The glide cylinders of the loop are separated by the distance $l=37 \mathrm{~nm}$. Using the Foreman-Eshelby expression (18), we see that the scale of elastic interaction between the loops in this case (the depth of the potential well similar to that shown in Fig. 10) is on the order of $-3 \mathrm{eV}$. After taking into account the possible weakening of interaction due to elastic anisotropy effects, we conclude that the binding energy, holding the two loops together, is on the order of $E_{b} \sim 2 \mathrm{eV}$. Given that $\exp \left(-E_{b} / k_{B} T_{0}\right) \sim 3 \times 10^{-16}$, it is not surprising to see that in experiment the loops stay together, migrating as two bound entities over a long period of time, exceeding the entire duration of experimental observation $\sim 80 \mathrm{~s}$. Figure 11 illustrates this behavior, showing the trajectories of the loops simulated by integrating the coupled Langevin equations, and comparing them with trajectories observed experimentally using in situ electron microscopy. In simulations and in observations we find a fairly similar scale of fluctuations for the position of the center of mass of the loops. Simulations also confirm that the strength of elastic interaction is sufficient for holding the loops together as bound entities over the entire interval of time spanned by the simulation $(\sim 20 \mathrm{~s})$. Figure 12 shows simulated trajectories of loops whose size and initial positions are similar to that observed using in situ electron microscopy and shown in Fig. 1. This case is more interesting and complex. The loop seen on the right $(\mathrm{R})$ is sufficiently far apart from the loop in the center $(\mathrm{C})$, and the binding energy between these two loops, evaluated using the Foreman-Eshelby formula (18), is on the order of $0.06 \mathrm{eV}$. Simulations show, in agreement with experiment, that this loop (R) is very weakly bound to the other two, and is able to move almost freely. We clearly see this trend already in the series of snapshots in Fig. 1 , and now this observation is confirmed directly by simulation. We see that the trajectory of loop $\mathrm{R}$ remains in the vicinity of the other two loops for no longer that $50 \mathrm{~s}$, and then loop R gets detached from the other two, and continues performing effectively free Brownian motion until the end of the time interval spanned by the simulation. 

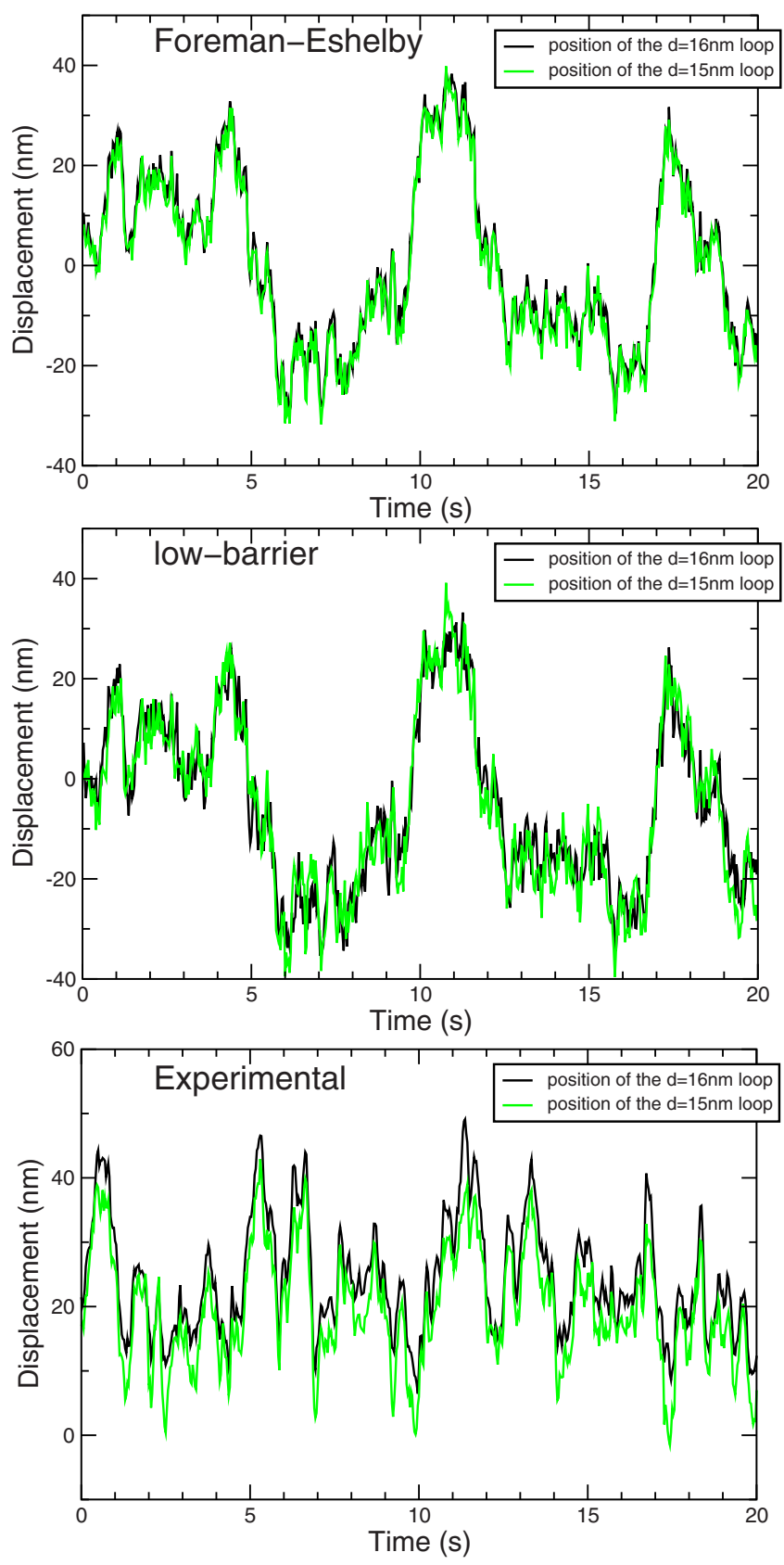

FIG. 11. (Color online) Comparison of trajectories of motion for two interacting $d=16 \mathrm{~nm}$ and $d=15 \mathrm{~nm}$ prismatic $\mathbf{b}=a / 2\langle 111\rangle$ dislocation loops migrating in pure iron at $T=650 \mathrm{~K}$. The glide cylinders of the loops are $37 \mathrm{~nm}$ apart. The top figure shows the trajectories of the two loops simulated using the Foreman-Eshelby expression (18) for the energy of interaction between the loops. The trajectories shown in the middle were simulated using Eq. (20). The figure at the bottom shows the trajectories of the loops observed experimentally using in situ electron microscopy.

On the other hand, the scale of elastic interactions binding loops $\mathrm{L}$ and $\mathrm{C}$ together is on the order of $0.3 \mathrm{eV}$, which is significantly greater than the energy of binding for loops $\mathrm{C}$ and R. Figure 12 shows that not only is this energy sufficient for capturing loop L, which comes in the field of view of the

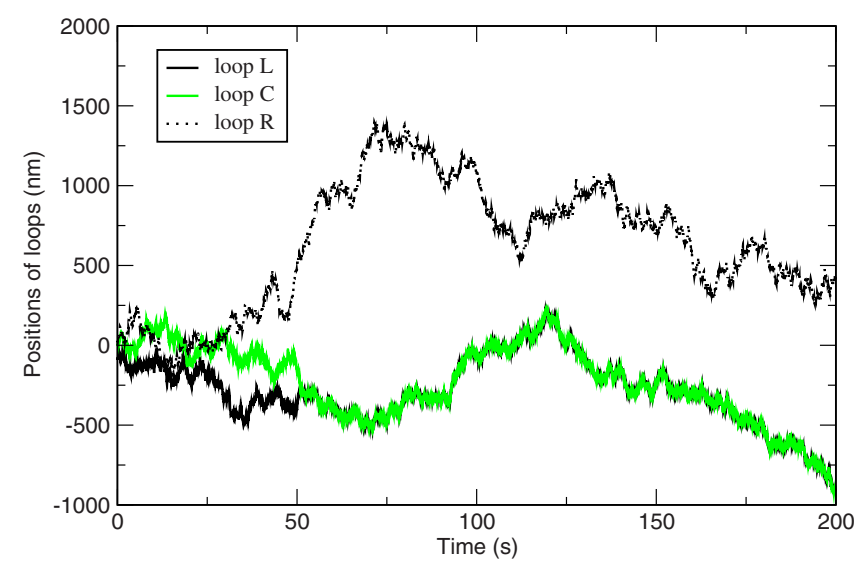

FIG. 12. (Color online) Simulated trajectories of Brownian motion for the three interacting dislocation loops shown in Fig. 1. Indexes L, C, and R refer to the loops seen in Fig. 1 on the left, in the center, and on the right. The trajectories were simulated using the coupled Langevin equations of motion for the loops [Eq. (9)] assuming that the energy of interaction between the loops is given by formula (20). Simulations were performed for $T_{0}=675 \mathrm{~K}$.

microscope from a distant part of the specimen, and appears only in the third snapshot shown in Fig. 1 but it is also sufficiently strong for binding the loops together over the entire interval of simulation spanning $200 \mathrm{~s}$. It may appear surprising that a binding energy as low as $0.3 \mathrm{eV}$ can actually hold the two migrating loops together over such a long interval of time. Indeed, the Arrhenius exponential factor for $T_{0}=675 \mathrm{~K}$ and $E_{b}=0.3 \mathrm{eV}$ is just $\exp \left(-E_{b} / k_{B} T\right) \approx 10^{-3}$, and the transition-state theory ${ }^{60,61}$ would predict that the two loops should break apart on the nanosecond time scale. However, in the present case the prefactor derived from a transition-state theory treatment ${ }^{60,61}$ does not apply, and instead the rate of escape by diffusion from a potential well is given by ${ }^{62}$

$$
\dot{r} \sim \frac{D}{W^{2}} \exp \left(-E_{b} / k_{B} T\right)
$$

where $D$ is the effective diffusion coefficient and $W$ is the width of the potential well, which in the case of elastic interaction is of the same order of magnitude as the effective size of interacting dislocation loops. For $D \sim 10^{3} \mathrm{~nm}^{2} \mathrm{~s}^{-1}$ and $W \sim 10 \mathrm{~nm}$, we find that the probability of the system of two bound loops falling apart per unit time equals $\dot{r} \sim 5.7$ $\times 10^{-2} \mathrm{~s}^{-1}$. In other words, the two loops bound by the relatively weak elastic forces are able to stay together as a single entity for up to $\sim 100 \mathrm{~s}$. A direct simulation extending over a much longer interval of time shows that for the case of three interacting loops illustrated in Fig. 12 the bound state of the two loops $\mathrm{L}$ and $\mathrm{C}$ remains stable on the time scale of $\sim 1000 \mathrm{~s}$, after which the loops separate. This relatively long time scale of stability does not contradict our initial estimate based on Eq. (21) since a direct Langevin simulation takes into account the effect of the shape of the potential well on the escape probability, not taken into account in formula (21) derived for the case of a square well. ${ }^{62}$ 
To quantify the argument given above, we consider a set of coupled Langevin equations for the two migrating and interacting loops,

$$
\begin{aligned}
& \frac{d z_{1}}{d t}=-\frac{D_{1}}{k_{B} T} \frac{\partial U\left(\left|z_{1}-z_{2}\right|\right)}{\partial z_{1}}+\sqrt{2 D_{1}} \xi_{1}(t), \\
& \frac{d z_{2}}{d t}=-\frac{D_{2}}{k_{B} T} \frac{\partial U\left(\left|z_{1}-z_{2}\right|\right)}{\partial z_{2}}+\sqrt{2 D_{2}} \xi_{2}(t) .
\end{aligned}
$$

Introducing new independent variables $z=z_{1}-z_{2}$ and $Z$ $=\left(D_{2} z_{1}+D_{1} z_{2}\right) /\left(D_{2}+D_{1}\right)$, we transform Eq. (22) as

$$
\begin{gathered}
\frac{d z}{d t}=-\frac{\left(D_{1}+D_{2}\right)}{k_{B} T} \frac{\partial U(|z|)}{\partial z}+\sqrt{2\left(D_{1}+D_{2}\right)} \xi_{z}(t), \\
\frac{d Z}{d t}=\sqrt{2 \frac{D_{1} D_{2}}{D_{1}+D_{2}}} \xi_{Z}(t),
\end{gathered}
$$

where both $\xi_{z}(t)$ and $\xi_{Z}(t)$ are random $\delta$-correlated functions of time $t$, i.e., $\overline{\xi_{z}(t) \xi_{z}\left(t^{\prime}\right)}=\delta\left(t-t^{\prime}\right)$ and $\overline{\xi_{Z}(t) \xi_{Z}\left(t^{\prime}\right)}=\delta\left(t-t^{\prime}\right)$. According to the second of the two Eqs. (23), the motion of the effective diffusion-weighted "center of mass" of the loops $Z(t)$ can be separated from the relative motion of the loops, described by their relative coordinate $z(t)$. This shows that the correlated motion of loops seen in Figs. 11 and 12 is the effect of trapping of loops by the attractive elastic interaction between the loops, which occurs in the moving frame of the effective diffusion-weighted center of mass for the system of two loops.

The position $Z(t)$ of the effective diffusion-weighted center of mass of the system of two loops, on the other hand, performs free Brownian motion entirely unaffected by the interaction between the loops. The diffusion coefficient for the position of the diffusion-weighted center of mass $Z(t)$, according to the second of the two Eqs. (23), is $D_{1} D_{2} /\left(D_{1}\right.$ $+D_{2}$ ). We note an interesting fact that this diffusion coefficient, in the limit where one of the diffusion coefficients $D_{1}$ or $D_{2}$ is significantly smaller than the other, equals the smallest of the two, irrespective of the strength of interaction between the loops. This conclusion appears to be general, for example, Brownian motion of a large ensemble of interacting defects is controlled by the slowest moving particle in the ensemble.

The loops can eventually separate from each other according to Eq. (21) but the time scale on which this separation occurs is fairly macroscopic. This conclusion agrees with experimental observations shown in Fig. 1, and many other observations of similar kind, which all exhibit extended intervals of correlated motion of loops. The fact that the loops become trapped and remain effectively immobile with respect to each other over long periods of time, is significant since this facilitates reactions between the loops, for example, the coalescence of loops, or the formation of extended rafts of loops, also observed experimentally. ${ }^{1-6}$

In this section, we showed that the relatively weak elastic interactions between migrating nanodislocation loops are able to facilitate the formation of bound long-lived configurations, where loops are trapped together and remain effectively stationary in the moving center of mass frame. The formation of these quasistable configurations increases the probability of reaction between the loops and provides a significant driving force for microstructural evolution, particularly in the limit of high irradiation dose rate.

\section{TRAPPING OF MIGRATING LOOPS BY VACANCY CLUSTERS}

In the previous section, we investigated the effect of trapping of two, or several, migrating dislocation loops by their mutual elastic fields, which occurs in the moving frame of the center of mass of the loops. In this section, we investigate a similar effect, observed for ion-beam irradiation conditions, ${ }^{4-6}$ where migrating dislocation loops are trapped, in the laboratory frame, by immobile vacancy clusters.

The assertion that trapping of migrating self-interstitial loops occurs as a result of interaction between a loop and vacancy clusters situated in the glide cylinder of the loop is at this point a hypothesis, stimulated by the observation that trajectories of motion of loops formed in electron- and ionirradiated iron are very different (see Figs. 2 and 3). If a vacancy, or a vacancy cluster, approaches a self-interstitial loop in the vicinity of its perimeter, the resulting annihilation reaction gives rise to the loop changing its shape, and the vacancy cluster disappearing. Similarly, the (repulsive) interaction between a loop and a vacancy cluster situated outside the glide cylinder for the loop does not explain trapping since the trajectories of migrating loops investigated for this case by Hudson et al. $^{26}$ resemble those shown in Fig. 2. The fairly long (up to $10 \mathrm{~s}$ ) time scales characterizing trapping of defects found in observations, are difficult to explain using the conventional transition-state theory argument ${ }^{60,61}$ that applies to processes occurring at atomic scale. Indeed, assuming an attempt frequency of $\nu^{*} \sim 10^{13} \mathrm{~Hz}$, for $T_{0}$ $=675 \mathrm{~K}$ we find that the binding energy required for trapping a loop at a lattice site for $\tau^{*} \sim 10 \mathrm{~s}$ is on the order of $E_{b} \sim k_{B} T_{0} \ln \left(\tau^{*} \nu^{*}\right) \approx 2 \mathrm{eV}$. This fairly high energy, comparable with the formation energy for a vacancy in iron, could, in principle, be attributed to interaction with complex stable configurations involving carbon or nitrogen impurities. ${ }^{63}$ Even after noting the slightly different levels of purity of the electron- and ion-irradiated samples, this still does not explain why these stable impurity complexes only form under ion irradiation, and do not form under electron irradiation. It is probably more natural to assume that ion irradiation, producing small vacancy clusters in the core of collision cascades, generates fine vacancy-cluster microstructures, interacting with, and trapping, the mobile self-interstitial dislocation loops.

The potential energy of interaction between a mobile loop and a vacancy-cluster defect is shown in Fig. 8. The energy scale characterizing interaction between the defects for the case shown in this figure is less than $0.9 \mathrm{eV}$. The shape and the energy scale of curves describing elastic interaction between a dislocation loop and an immobile vacancy cluster 


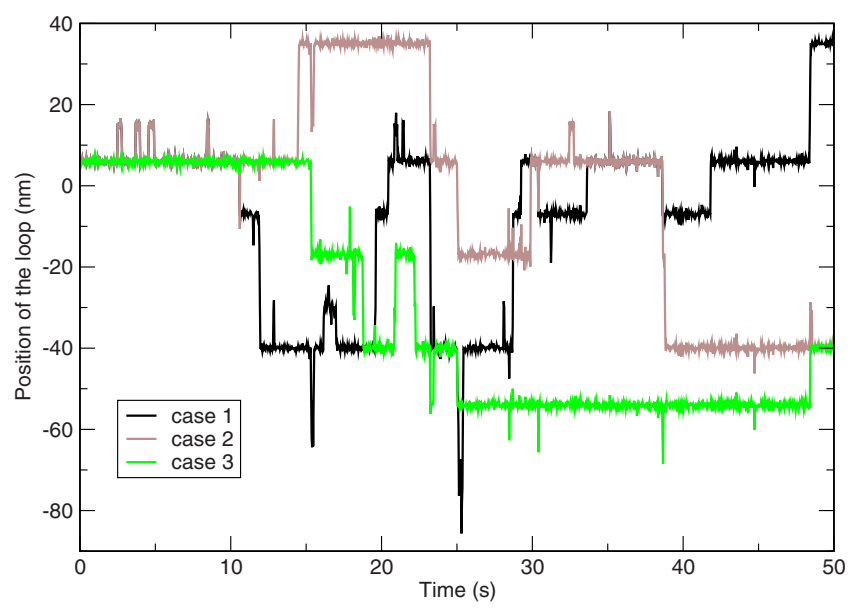

FIG. 13. (Color online) Three realizations for trajectories of motion for a $d=6 \mathrm{~nm}$ prismatic $\mathbf{b}=a / 2\langle 111\rangle$ dislocation loop migrating in pure iron at $T=675 \mathrm{~K}$ and interacting with statistically distributed vacancy clusters. The diffusion coefficient for the migrating loop is $D=1875 \mathrm{~nm}^{2} \mathrm{~s}^{-1}$. The three realizations of trajectories correspond to three different realization of the potential-energy landscape, corresponding to three statistical realizations of the distribution of vacancy clusters in the material.

varies, according to Eq. (10), as a function of the size of the loop, and the position of the vacancy cluster within the glide cylinder of the loop. Hence it is expected that a nanoscale loop migrating in a crystal containing vacancy defects would move under the action of a field of randomly distributed vacancy defects, which themselves may have randomly varying size. The fact that the location and the size of vacancy defects fluctuate causes the "external" interaction potential felt by a migrating self-interstitial loop to be a fluctuating function of the position of the loop. For example, we can represent the function $U(z)$ for an individual loop, described by a single Langevin equation and by a set of potential wells of varying depth and width, distributed along the trajectory of migration for the loop.

Figure 13 shows three examples of trajectories of migration of a single $d=6 \mathrm{~nm}$ prismatic dislocation loop migrating in pure iron and interacting with statistically distributed vacancy clusters. The effective potential energy of interaction, corresponding to case 3 shown in Fig. 13, is shown in Fig. 14. The simulated trajectories of migration of the loop closely resemble those shown in Figs. 3 and 15, and other similar trajectories observed earlier. ${ }^{4,6,41}$ The similarity between the fairly characteristic shape of trajectories found in simulations and in experimental observations, and the similarity between the time scales characterizing the trapping events, suggest that it is the elastic interaction between the loops and vacancy clusters formed under cascade irradiation that likely gives rise to the unusual statistics of motion (hopping) of loops observed by in situ electron microscopy for ion-irradiated materials. Such vacancy clusters remain almost invisible in electron microscope images. ${ }^{41}$ The statistics of trapping and detrapping events, and transitions between the trapping sites seen in Fig. 13 is in good agreement with

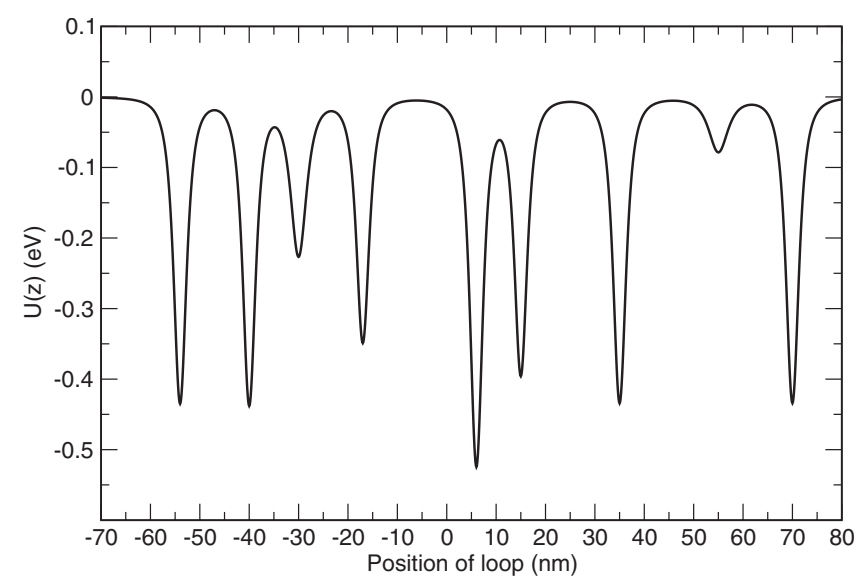

FIG. 14. The effective potential energy $U(z)$ of interaction between a mobile prismatic dislocation loop and statistically distributed vacancy clusters. The statistical realization of potential energy shown in this figure corresponds to the trajectory labeled as case 3 in Fig. 13.

the estimated residence times given by Eq. (21). We therefore conclude that it is the combined effect of elastic interaction between the loops and the vacancy clusters, and Brownian migration of the loops, that is likely responsible for the unusual features of migration of loops in ionirradiated iron shown in Figs. 3 and 15. Effects associated with elastic long-range interaction between migrating loops themselves are responsible for the correlated motion of loops frequently observed in high-energy electron irradiated iron, which is shown in Fig. 1.

\section{CONCLUSIONS}

In this paper, we developed a Langevin dynamics model and showed that the model is able to match real-time in situ electron microscope observations of dynamics of migration and interaction between mobile nanoscale defects. We find

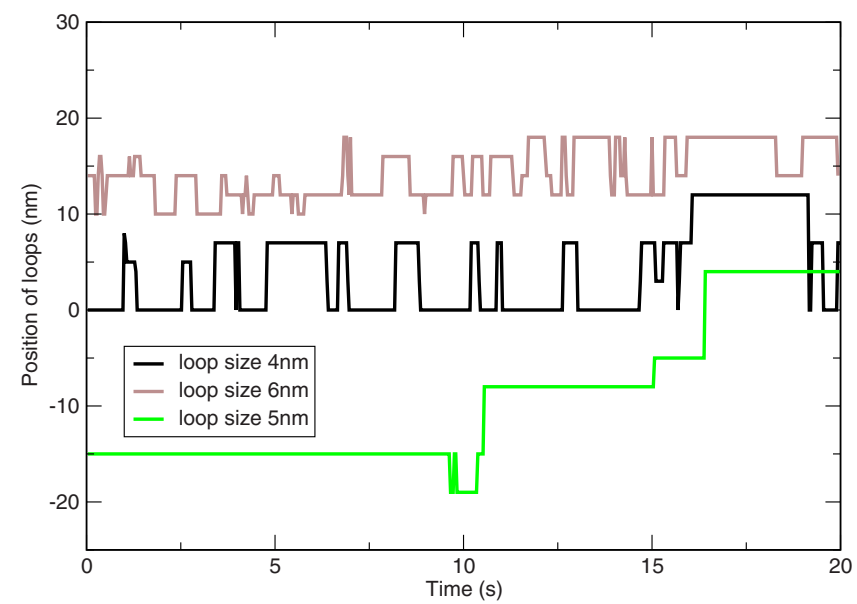

FIG. 15. (Color online) Examples of experimentally observed trajectories of migration for prismatic $a / 2\langle 111\rangle$ dislocation loops of various size in pure iron irradiated with $150 \mathrm{keV} \mathrm{Fe}^{+}$ions to a dose of $\sim 0.65 \mathrm{dpa}$. The temperature of the specimen is $673 \mathrm{~K}$. 
that diffusion of defects combined with effects of elastic interactions gives rise to the occurrence of new modes of microstructural evolution, involving defect trapping and correlated motion of defects, which in turn strongly influence the rates of reaction and coalescence between the defects. The Langevin dynamics approach described above is able to model the dynamics of motion of radiation defects on realistic time scales (10-1000 s), matching those of experimental observations, as opposed to MD simulations that are only able to address time scales many orders of magnitude shorter.

The Langevin dynamics model appears particularly suitable for treating the effects of interaction between the defects, which so far have proved difficult to include in kinetic Monte Carlo simulations while at the same time offering advantages related to the computational implementation of the algorithm. The method will likely prove effective for interpreting experimental data on microstructures formed by ion-beam irradiation of materials, where the high-dose-rate effects and various phenomena related to long-range interac- tions between the defects, have so far proved difficult to address using other modeling techniques and algorithms.

\section{ACKNOWLEDGMENTS}

One of the authors (S.L.D.) gratefully acknowledges stimulating discussions with V. V. Bulatov, and hospitality of C. H. Woo at Hong-Kong Polytechnic University, where part of this work was completed. This work was supported by the UK EPSRC, by EURATOM, and by EFDA staff mobility program. The views and opinions expressed herein do not necessarily reflect those of the European Commission. We also acknowledge support from Grant-in-Aid for Scientific Research (Grant No. 21560686) and Priority Assistance for the Formation of Worldwide Renowned Centers of Research-The Global COE Program (Project: Center of Excellence for Advanced Structural and Functional Materials Design) from the Ministry of Education, Sport, Culture, Science, and Technology of Japan.
${ }^{1}$ K. Arakawa, M. Hatanaka, E. Kuramoto, K. Ono, and H. Mori, Phys. Rev. Lett. 96, 125506 (2006).

${ }^{2}$ K. Arakawa, K. Ono, M. Isshiki, K. Mimura, M. Uchikoshi, and H. Mori, Science 318, 956 (2007).

${ }^{3} \mathrm{~K}$. Arakawa, K. Ono, and H. Mori, in Electron Microscopy and Multiscale Modelling, edited by A. Avilov et al., AIP Conf. Proc. No. 999 (AIP, New York, 2008), pp. 66-78.

${ }^{4}$ Z. Yao, M. Hernández-Mayoral, M. L. Jenkins, and M. A. Kirk, Philos. Mag. 88, 2851 (2008).

${ }^{5}$ M. Hernández-Mayoral, Z. Yao, M. L. Jenkins, and M. A. Kirk, Philos. Mag. 88, 2881 (2008).

${ }^{6}$ M. L. Jenkins, Z. Yao, M. Hernández-Mayoral, and M. A. Kirk, J. Nucl. Mater. 389, 197 (2009).

${ }^{7}$ S. L. Dudarev, R. Bullough, and P. M. Derlet, Phys. Rev. Lett. 100, 135503 (2008); S. L. Dudarev, P. M. Derlet, and R. Bullough, J. Nucl. Mater. 386-388, 45 (2009).

${ }^{8}$ S. P. Fitzgerald and Z. Yao, Philos. Mag. Lett. 89, 581 (2009).

${ }^{9}$ S. P. Fitzgerald and S. L. Dudarev, Proc. R. Soc. London, Ser. A 464, 2549 (2008); J. Nucl. Mater. 386-388, 67 (2009).

${ }^{10}$ B. D. Wirth, G. R. Odette, D. Maroudas, and G. E. Lucas, J. Nucl. Mater. 244, 185 (1997); 276, 33 (2000).

${ }^{11}$ D. J. Bacon, F. Gao, and Yu. N. Osetsky, J. Comput.-Aided Mater. Des. 6, 225 (1999).

${ }^{12}$ E. Kuramoto, J. Nucl. Mater. 276, 143 (2000).

${ }^{13}$ K. Morishita, T. Diaz de la Rubia, E. Alonso, N. Sekimura, and N. Yoshida, J. Nucl. Mater. 283-287, 753 (2000).

${ }^{14}$ J. Marian, B. D. Wirth, A. Caro, B. Sadigh, G. R. Odette, J. M. Perlado, and T. Diaz de la Rubia, Phys. Rev. 65, 144102 (2002).

${ }^{15}$ Yu. N. Osetsky, D. J. Bacon, A. Serra, B. N. Singh, and S. I. Golubov, Philos. Mag. 83, 61 (2003).

${ }^{16}$ C.-H. Woo and B. N. Singh, Philos. Mag. 65, 889 (1992).

${ }^{17}$ P. B. Hirsch, A. Howie, R. Nicholson, D. W. Pashley, and M. J. Whelan, Electron Microscopy of Thin Crystals (Krieger, Malabar, FL, 1977).

${ }^{18}$ L. M. Peng, S. L. Dudarev, and M. J. Whelan, High-Energy
Electron Diffraction and Microscopy (Oxford University Press, Oxford, 2004).

${ }^{19}$ Y. Serruys, M.-O. Ruault, P. Trocellier, S. Miro, A. Barbu, L. Boulanger, O. Kaitasov, S. Henry, O. Leseigneur, P. Trouslard, S. Pellegrino, and S. Vaubaillon, C. R. Phys. 9, 437 (2008).

${ }^{20}$ D. Iracane, P. Chaix, and A. Alamo, C. R. Phys. 9, 445 (2008).

${ }^{21}$ A. Möslang, C. R. Phys. 9, 457 (2008).

${ }^{22}$ D. J. Ward and S. L. Dudarev, Mater. Today 11 (12), 46 (2008).

${ }^{23}$ J. E. Westmoreland, J. A. Sprague, F. A. Smidt, Jr., and P. R. Malmberg, Radiat. Eff. Defects Solids 26, 1 (1975).

${ }^{24}$ J. E. Westmoreland, P. R. Malmberg, J. A. Sprague, and F. A. Smidt, J. Vac. Sci. Technol. 12, 511 (1975).

${ }^{25}$ O. W. Holland, J. Narayan, and D. Fathy, ORNL Report No. CONF-840760-12, 1984 (unpublished).

${ }^{26}$ T. S. Hudson, S. L. Dudarev, and A. P. Sutton, Proc. R. Soc. London, Ser. A 460, 2457 (2004).

${ }^{27}$ T. S. Hudson, S. L. Dudarev, M.-J. Caturla, and A. P. Sutton, Philos. Mag. 85, 661 (2005).

${ }^{28}$ M. Pelfort, Yu. N. Osetsky, and A. Serra, Philos. Mag. Lett. 81, 803 (2001).

${ }^{29}$ M. A. Puigvi, Yu. N. Osetsky, and A. Serra, Mater. Sci. Eng., A 365, 101 (2004).

${ }^{30}$ T. Opplestrup, V. V. Bulatov, G. H. Gilmer, M. H. Kalos, and B. Sadigh, Phys. Rev. Lett. 97, 230602 (2006).

${ }^{31}$ E. Martinez, J. Marian, M. H. Kalos, and J. M. Perlado, J. Comput. Phys. 227, 3804 (2008).

${ }^{32}$ J. Zinn-Justin, Quantum Field Theory and Critical Phenomena (Clarendon, Oxford, 1993), Chap. 4.

${ }^{33} \mathrm{H}$. Risken, The Fokker-Planck Equation (Springer, Berlin, 1996).

${ }^{34}$ P. M. Derlet, D. Nguyen-Manh, and S. L. Dudarev, Phys. Rev. B 76, 054107 (2007).

${ }^{35}$ S. L. Dudarev, C. R. Phys. 9, 409 (2008).

${ }^{36}$ T. D. de la Rubia, Annu. Rev. Mater. Sci. 26, 613 (1996).

${ }^{37}$ S. L. Dudarev, P. M. Derlet, and C. H. Woo, Nucl. Instrum. 
Methods B256, 253 (2007).

${ }^{38}$ Y. Satoh, H. Matsui, and T. Hamaoka, Phys. Rev. B 77, 094135 (2008).

${ }^{39}$ J. A. Hinks, Nucl. Instrum. Methods Phys. Res. B 267, 3652 (2009).

${ }^{40}$ S. L. Dudarev, J.-L. Boutard, R. Lässer, M. J. Caturla, P. M. Derlet, M. Fivel, C.-C. Fu, M. Y. Lavrentiev, L. Malerba, M. Mrovec, D. Nguyen-Manh, K. Nordlund, M. Perlado, R. Schäublin, H. Van Swygenhoven, D. Terentyev, J. Wallenius, D. Weygand, and F. Willaime, J. Nucl. Mater. 386-388, 1 (2009).

${ }^{41}$ M. R. Gilbert, Z. Yao, M. A. Kirk, M. L. Jenkins, and S. L. Dudarev, J. Nucl. Mater. 386-388, 36 (2009).

${ }^{42}$ S. L. Dudarev and P. M. Derlet, J. Phys.: Condens. Matter 17, 7097 (2005); 19, 239001 (2007); P. M. Derlet and S. L. Dudarev, Prog. Mater. Sci. 52, 299 (2007).

${ }^{43}$ S. L. Dudarev, J. Nucl. Mater. 307-311, 881 (2002).

${ }^{44}$ S. L. Dudarev, Philos. Mag. 83, 3577 (2001).

${ }^{45}$ M. R. Gilbert, S. L. Dudarev, P. M. Derlet, and D. G. Pettifor, J. Phys.: Condens. Matter 20, 345214 (2008).

${ }^{46}$ W. T. Coffey, Y. P. Kalmykov, and J. T. Waldron, The Langevin Equation (World Scientific, Singapore, 1996).

${ }^{47}$ B. U. Felderhof, J. Phys. A 11, 929 (1978).

${ }^{48}$ J. M. Deutch and I. Oppenheim, J. Chem. Phys. 54, 3547
(1971).

${ }^{49}$ T. J. Murphy and J. L. Aguirre, J. Chem. Phys. 57, 2098 (1972).

${ }^{50}$ K. M. Hong and J. Noolandi, Surf. Sci. 75, 561 (1978).

${ }^{51}$ M. Doi and S. F. Edwards, The Theory of Polymer Dynamics (Clarendon, Oxford, 1986), Chap. 3, pp. 46-90.

${ }^{52}$ J. Baštecká and F. Kroupa, Czech. J. Phys. B 14, 443 (1964).

${ }^{53}$ Handbook of Mathematical Functions with Formulas, Graphs, and Mathematical Tables, edited by M. Abramowitz and I. A. Stegun (Dover, New York, 1972).

${ }^{54}$ C. K. Ong, Phys. Status Solidi B 111, 331 (1982).

${ }^{55}$ Yu. N. Osetsky, A. G. Mikhin, and A. Serra, Philos. Mag. A 72, 361 (1995).

${ }^{56}$ J. P. Hirth and J. Lothe, Theory of Dislocations, 2nd ed. (Wiley, New York, 1982).

${ }^{57}$ A. J. E. Foreman and J. D. Eshelby, AERE Report No. 4170, 1962 (unpublished).

${ }^{58}$ R. S. Barnes, J. Phys. Soc. Jpn. 18, Suppl. III, 305 (1963).

${ }^{59}$ J. R. Willis, Q. J. Mech. Appl. Math. 18, 419 (1965).

${ }^{60}$ G. H. Vineyard, J. Phys. Chem. Solids 3, 121 (1957).

${ }^{61}$ R. Landauer and J. A. Swanson, Phys. Rev. 121, 1668 (1961).

${ }^{62}$ B. U. Felderhof, Physica A 387, 39 (2008).

${ }^{63}$ C. C. Fu and F. Willaime, C. R. Phys. 9, 335 (2008). 